Research Article

\title{
Data Transfer Schemes in Rotorcraft Fluid-Structure Interaction Predictions
}

\author{
Young H. You, Deokhwan Na, and Sung N. Jung \\ Department of Aerospace Information Engineering, Konkuk University, Seoul 143-701, Republic of Korea \\ Correspondence should be addressed to Sung N. Jung; snjung@konkuk.ac.kr
}

Received 19 September 2017; Accepted 4 January 2018; Published 14 March 2018

Academic Editor: Vaios Lappas

Copyright ( 2018 Young H. You et al. This is an open access article distributed under the Creative Commons Attribution License, which permits unrestricted use, distribution, and reproduction in any medium, provided the original work is properly cited.

\begin{abstract}
For a CFD (computation fluid dynamics)/CSD (computational structural dynamics) coupling, appropriate data exchange strategy is required for the successful operation of the coupling computation, due to fundamental differences between CFD and CSD analyses. This study aims at evaluating various data transfer schemes of a loose CFD/CSD coupling algorithm to validate the higher harmonic control aeroacoustic rotor test (HART) data in descending flight. Three different data transfer methods in relation to the time domain airloads are considered. The first (method 1) uses random data selection matched with the timewise resolution of the CSD analysis whereas the last (method 2) adopts a harmonic filter to the original signals in CFD and CSD analyses. The second (method 3) is a mixture of the two methods. All methods lead to convergent solutions after a few cycles of coupling iterations are marched. The final converged solutions for each of the data transfer methods are correlated with the measured HART data. It is found that both method 1 and method 2 exhibit nearly identical results on airloads and blade motions leading to excellent correlations with the measured data while the agreement is less satisfactory with method 3. The reason of the discrepancy is identified and discussed illustrating CFD-/CSD-coupled aeromechanics predictions.
\end{abstract}

\section{Introduction}

Recently, with the advancement of modern software skills and innovative computer hardware technologies, mathematically challenging problems such as rotorcraft blade vortex interaction-(BVI-) induced noise and vibration have been tackled in a level that no one anticipated previously. One of the major breakthroughs in this regard is reached with the introduction of CFD method for loads and vibration prediction of a rotor. The classic CSD approach is combined with a CFD code in a form of loose coupling such that the blade elastic motions computed using CSD method and the aerodynamic forces and moments obtained using CFD method are exchanged at specified instant of time. This CFD/CSD coupling algorithm has been originally devised by Tung et al. [1] for simple lift coupling, developed separately by Beaumier [2] for lift and pitching moment coupling and later finalized by Potsdam et al. [3] using the delta airloads technique leading to close agreement with the measured data of UH-60A rotor. The CFD/CSD approach enables one to benefit the broad spectrum of CSD analysis power with the aid of first-principle-based CFD analysis capability, with the cost of computational efficiency. Even though the prediction capability has been improved drastically with CFD/CSD approach, there is no standard protocol or straightforward methodology for transferring data between CFD and CSD codes where significant gaps exist in solution methods and grid resolutions.

For rotor prediction capability, acquiring highly reliable test data plays crucial roles for cross-validating analytical results and implementing mathematical models to enhance physical understanding of an engineering phenomenon. Among other cases, HART data [4] offer unique opportunity to exploit and challenge high-frequency BVI-intensive signals in any advanced rotorcraft analysis system. The HART experiment was performed in the open-jet anechoic chamber of the German-Dutch wind tunnel (DNW) in 1994. The goal was to measure rotor loads, blade motions, acoustic signature, and flow fields in various flight conditions, with and without higher harmonic control (HHC) pitch control inputs. The 
TABLE 1: Comparison of instrumentations for airloads and structural loads.

\begin{tabular}{lcc}
\hline & HART & HART II \\
\hline Number of pressure transducers & 124 & 51 \\
Number of measured air stations & $3(r / R=0.75,0.87,0.97)$ & $1(r / R=0.87)$ \\
Number of strain gages & $34(13$ flap, 12 lag, and 9 torsion $)$ & $6(3$ flap, 2 lag, and 1 torsion) \\
\hline
\end{tabular}

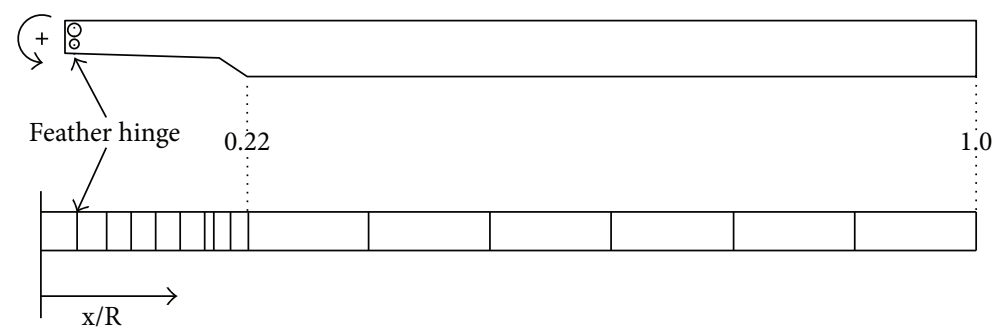

(a) Finite element model

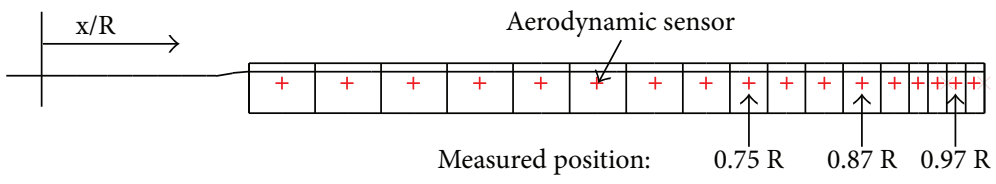

(b) Aerodynamic model

FIGURE 1: HART blade models used for CSD analysis.

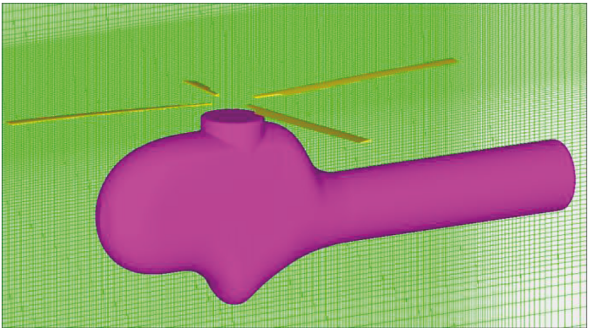

Figure 2: Computational grids used for CFD analysis.

international collaborative research results in a remarkable success [5], particularly obtaining high-precision test data set, advancing the smart rotor concept, gaining detailed knowledge of BVI phenomenon and its reduction mechanism due to the HHC technique, and leading to a follow-on test program HART II in 2001 [6]. A number of validation activities of HART rotor have been reported in the literature [7-11]. However, the published records are significantly less in volume as opposed to the postdecessor program HART II where the measured data have been extensively validated by researchers worldwide $[12,13]$. Furthermore, very limited work has been carried out via CFD/CSD coupling except the work of Lim et al. [9] where a significant improvement on airloads prediction is reached over the conventional CSD approaches. The effect of fuselage is however neglected in the analysis of Lim et al. [9] which may lead to a phase shift problem on airloads along with the underprediction of BVI peak oscillations as observed by Jung et al. [14]. In addition, the update of the blade structural properties obtained by the recent measurement activity $[15,16]$ is lacking in most of the published works.

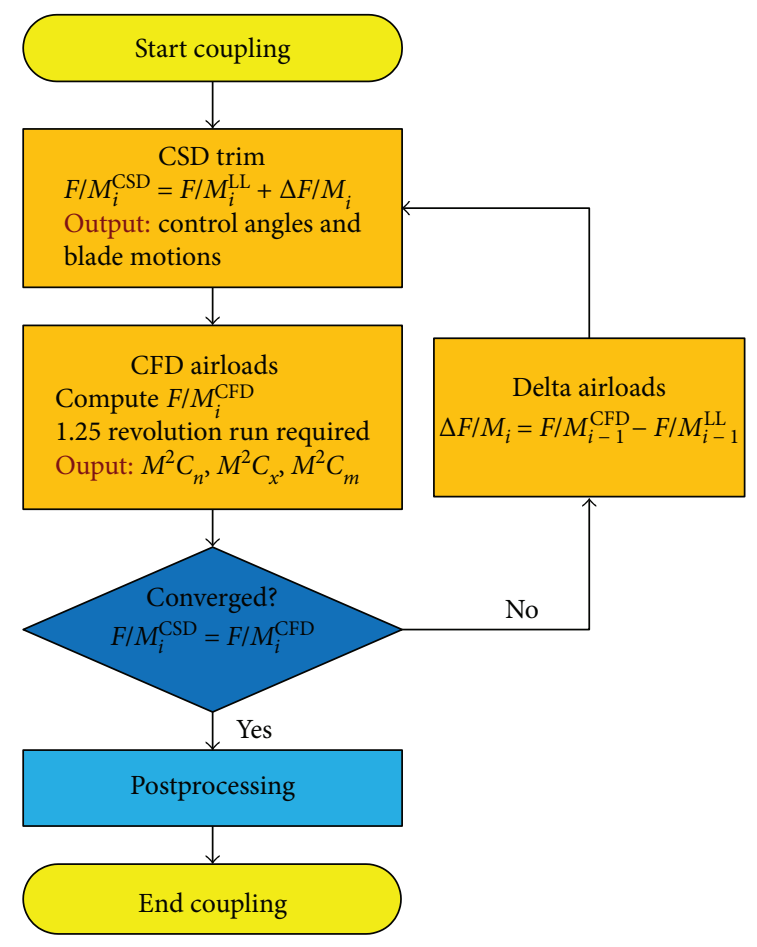

FIGURE 3: Flow diagrams for a loose CFD/CSD coupling approach.

The present study aims at conducting a refined computation using CFD/CSD coupling for HART rotor in descent flight. A three-dimensional (3D), compressible RANS (Reynolds averaged Navier-Stokes) solver is employed as a CFD code. Considering the missing gap in the literature associated with the data exchange algorithm between CFD and CSD codes, this study is focused to investigate several data transfer 


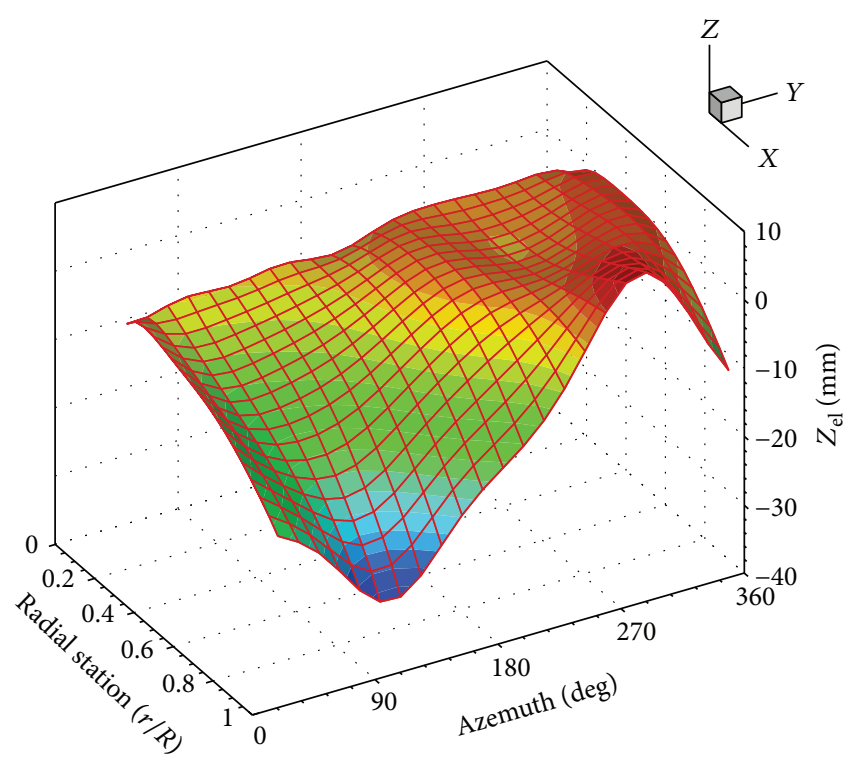

(a) Blade flap displacement

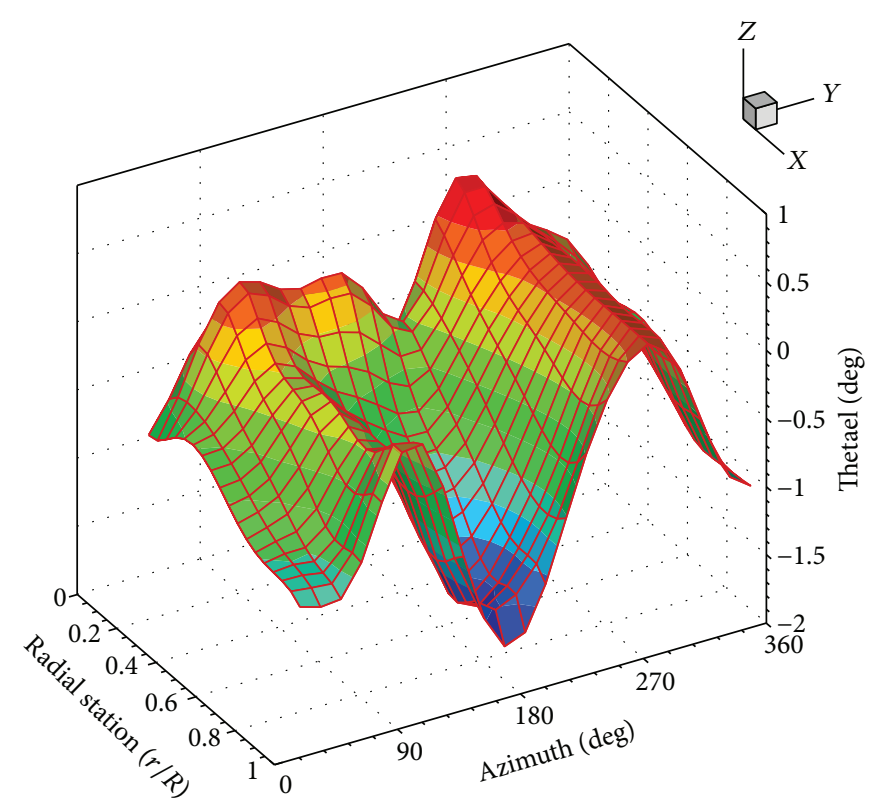

(b) Blade twist deformation

FIGURE 4: Blade elastic motions interpolated from CSD results.

TABLE 2: Summary of data synthesis methods.

\begin{tabular}{lccc}
\hline & Method 1 & Method 2 & Method 3 \\
\hline CFD airloads (timewise) & Every $5 \operatorname{deg}(\Delta \psi=5 \mathrm{deg})$ & Every $5 \operatorname{deg}(\Delta \psi=5 \mathrm{deg})$ & Low-pass filtered (up to 10/rev) \\
CSD airloads (timewise) & Every $5 \operatorname{deg}(\Delta \psi=5 \mathrm{deg})$ & Low-pass filtered (up to 10/rev) & Low-pass filtered (up to 10/rev) \\
Spanwise airloads (CFD \& CSD) & Cubic spline & Cubic spline & Cubic spline \\
Blade motions (spanwise) & Polynomial with $n$th order & Polynomial with $n$th order & Polynomial with $n$th order \\
Blade motions (timewise) & Fourier series with $m$ th & Polynomial with $m$ th & Polynomial with $m$ th \\
& components & components & components \\
\hline
\end{tabular}

schemes in a loose coupling approach. Three different methods classified associated with the time domain airloads are examined. The first uses specified timewise data selection matched with the CSD analysis whereas the last adopts a harmonic filter to the raw data. The second one is a mixture of both methods. The accuracy of the final converged solutions for each of the data transfer schemes is evaluated by comparison with the measured HART data which include section airloads, blade elastic motions, and structural moments of the rotor. In addition, the newly measured blade properties are used for more realistic analysis.

\section{HART Experiment}

The HART rotor was tested at the DNW by an international joint team in 1994 [4]. A four-bladed, 40\% Mach-scaled hingeless BO-105 model with $2 \mathrm{~m}$ radius and $0.121 \mathrm{~m}$ chord length is used for the test. The blade is constructed of Eglass spar and skin and has a rectangular planform with a modified NACA 23012 airfoil with trailing-edge tab. The test condition considered in the present study is a descending forward flight with an advance ratio $\mu=0.15$, shaft tilt angle $\alpha_{s}=4.5 \mathrm{deg}$ aft (after the wall correction), and thrust level $C_{T}=0.0044$. The rotor is trimmed to match the target values

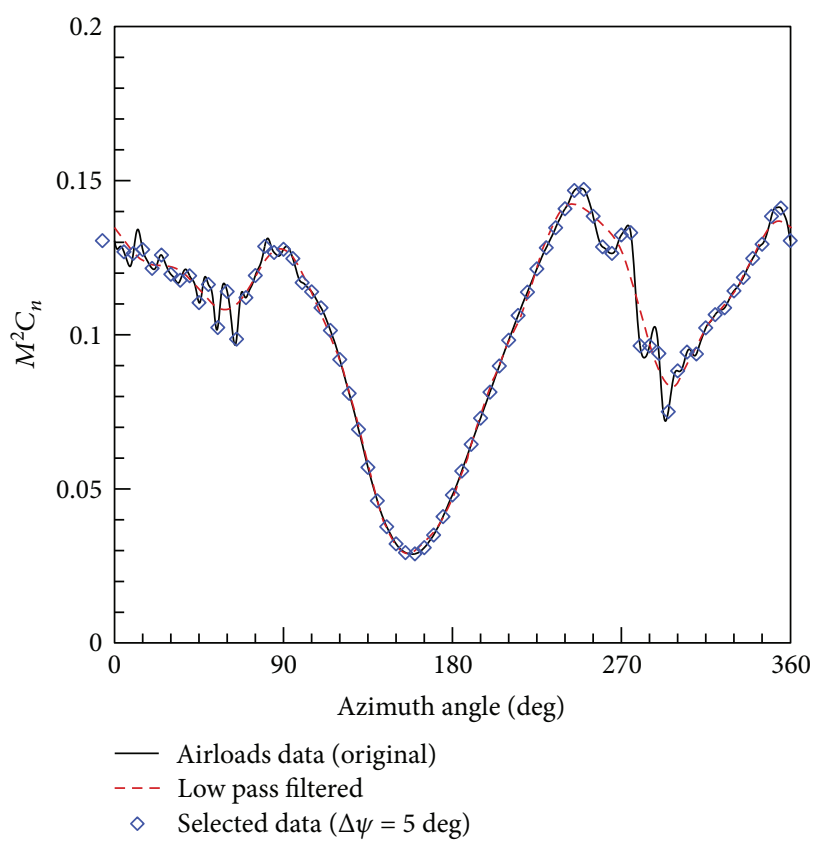

Figure 5: Comparison of data selection schemes on airloads signals. 


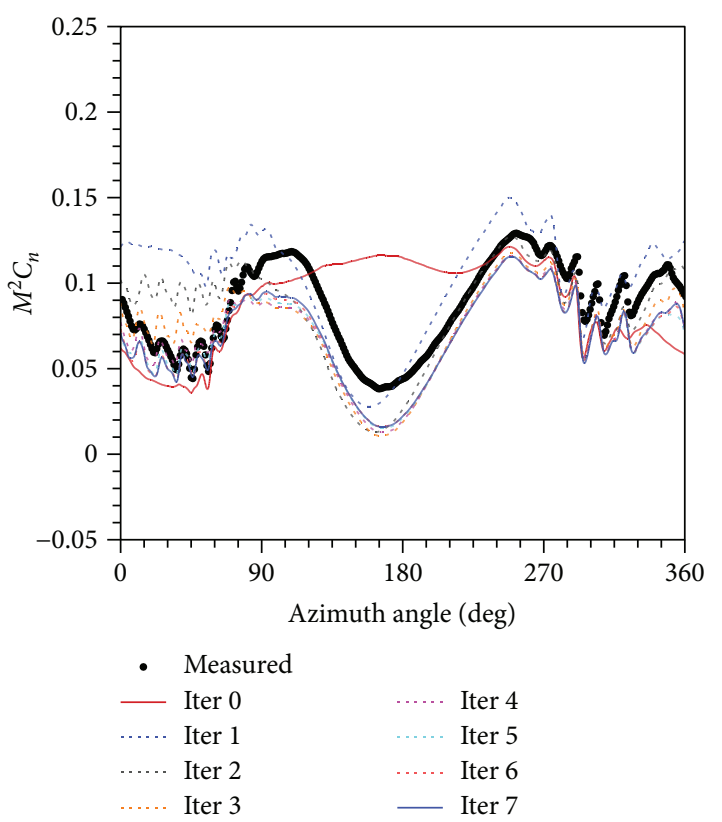

(a) Method 1

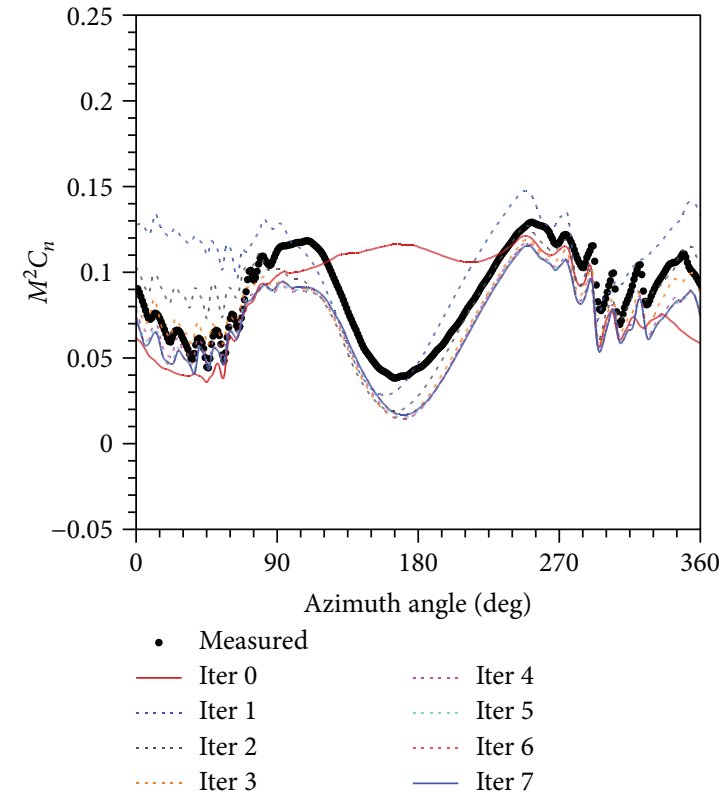

(b) Method 2

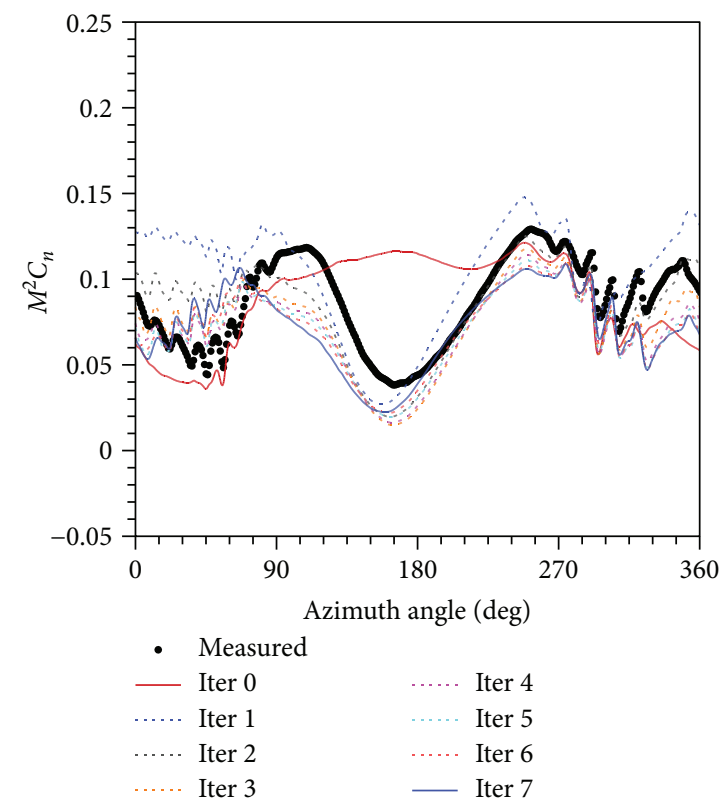

(c) Method 3

Figure 6: Comparison of convergence on section airloads $M^{2} C_{n}$ at $r / R=0.87$ with coupling iterations.

for the thrust, hub roll, and pitching moment as $3100 \mathrm{~N}$, 11.2 N-m, and $-20 \mathrm{~N}-\mathrm{m}$, respectively. The sign conventions used are positive when the advancing side goes up and a pitch-up is induced for the moments. The pressure measurements are made at three radial stations $(r / R=0.75,0.87$, and 0.97) of the reference blade ( $\mathrm{H} 1 \mathrm{Y})$ for the complete pressure distribution along the blade chord. A total of 32 strain gauges are attached on the blade surface, distributed between $r / R=0.14$ and 0.83 , to measure the structural loads as well as the elastic deformation of the blade. An alternative optical technique was used to measure the blade tip motions. It is noted that HART rotor allows wider spectrum for airloads and structural load data than the follow-on program HART II (see Table 1). The blade structural properties are measured recently using the original set of blades tested in the wind tunnel $[15,16]$, and these are implemented in the present analysis.

\section{Analysis Methodologies}

A loose CFD/CSD coupling is employed to validate the measured HART data. To this end, a CSD analysis code CAMRAD II [17] is combined with a RANS flow solver KFLOW 


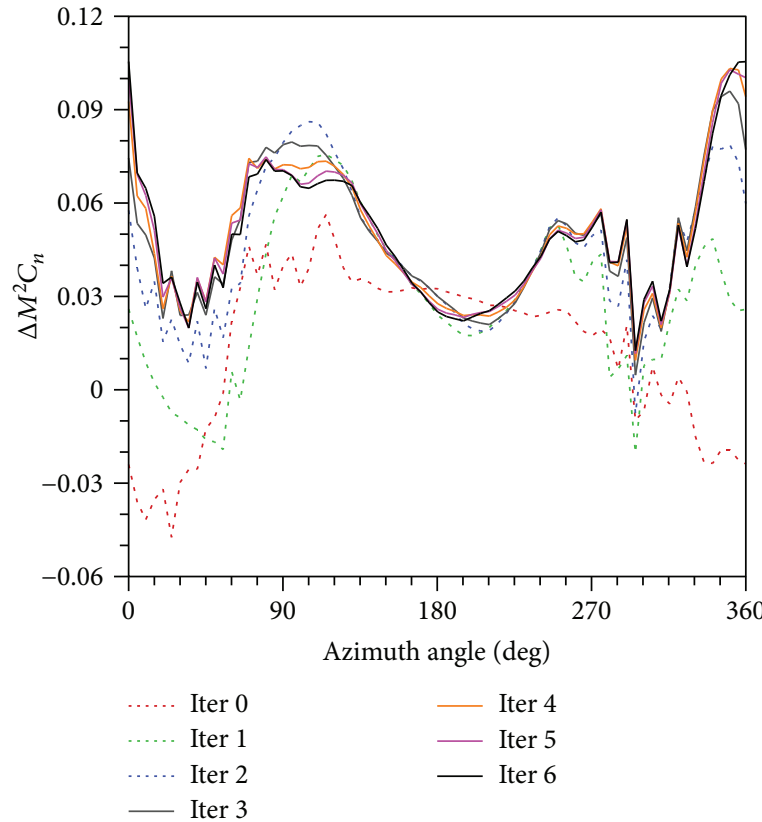

(a) Method 1

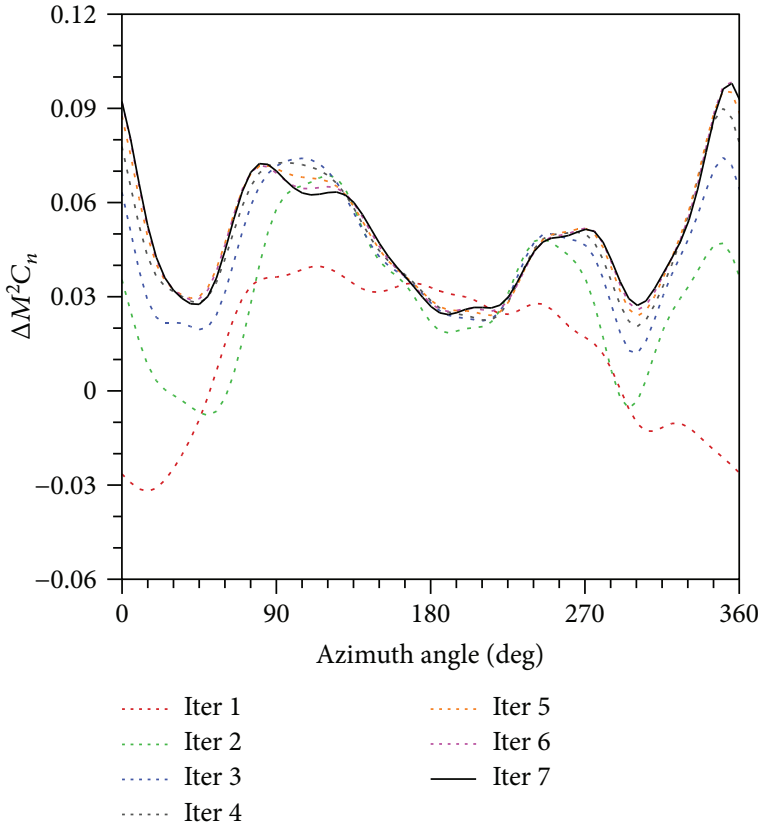

(b) Method 2

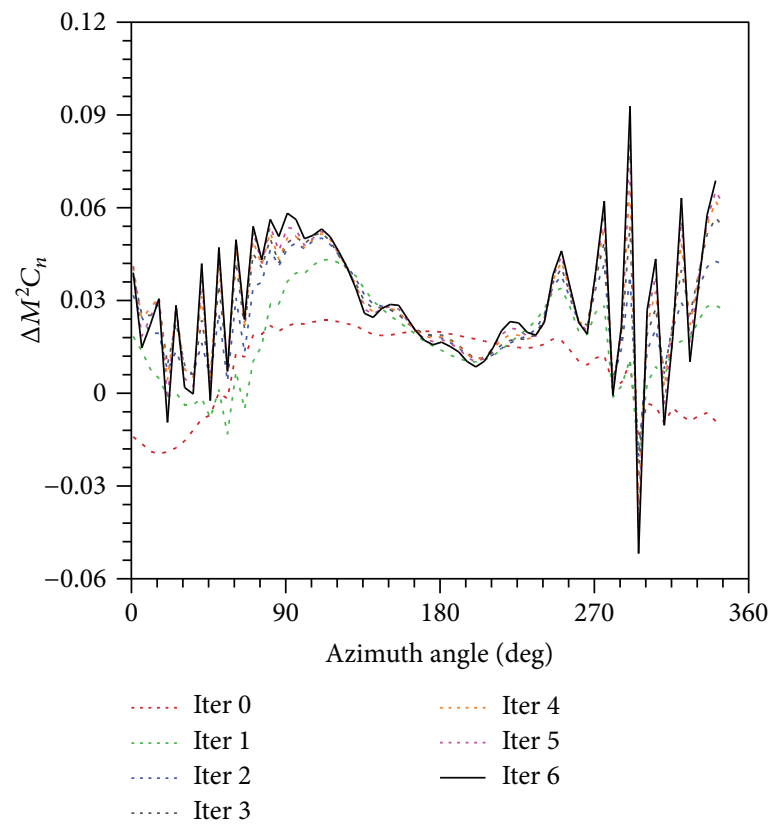

(c) Method 3

Figure 7: Comparison of convergence on delta airloads $\Delta M^{2} C_{n}$ at $r / R=0.87$ with coupling iterations.

[18]. The essential features and modeling details adopted in the present approach are summarized in this section.

3.1. CSD Approach. CAMRAD II is a comprehensive aeromechanical analysis tool that is characterized by multibody dynamics, nonlinear finite elements, and various level of rotorcraft aerodynamics [17]. For the structural analysis, the blade motion is composed of the rigid body motion and the elastic deformation. The rigid body motion describes the motion of one end of a beam element, and the elastic motion is measured relative to the rigid motion. The beam elements are represented by 6 degrees of freedom (DOF) for the rigid motion and $9 \mathrm{DOF}$ for the elastic motion (3 axial, 2 flap, 2 lag, and 2 torsion) that results in a 15 DOF for each beam finite element. The aerodynamic model is based on the ONERA-EDLIN unsteady airfoil theory combined with C81 airfoil table look-up. For the vortex wake model, the free wake geometry is used to compute the nonuniform-induced inflow around the rotor disk. The formation of the tip vortices is modeled using a free rolled-up wake model. The rolled-up wake model is based on the feature that a tip vortex forms at the blade tip. Both single and 
dual peak models are available considering the distribution of bound circulation peaks over the blade length.

In the present analysis, the blade structure is modeled using 15 beam finite elements with the discretization of finer elements in the blade inboard portion to counter a large variation in the structural properties of the blade, as can be seen in Figure 1(a). The airfoil blade region is divided into 17 nonuniform aerodynamic panels with finer lengths toward the blade tip, as shown in Figure 1(b). Specifically, the center of the aerodynamic panels is aligned to coincide with the measured airloads stations to minimize the discretization error.

3.2. CFD Approach. A 3D compressible flow solver KFLOW [18] is used for the CFD analysis. The KFLOW is a structured, parallelized multiblock, RANS solver that can compute time-accurate moving body problems. A second-order accurate, dual-time stepping scheme combined with a diagonalized alternating-directional implicit method is used to compute the unsteady flow fields around a rotor. The inviscid fluxes are calculated using the fifth-order weighted essentially nonoscillatory (WENO) scheme, while the central differencing technique is applied to the viscous fluxes. The $k-\omega$ Wilkox-Durbin (WD+) scheme is adopted for the turbulence model. The characteristic boundary conditions using the Riemann invariant are applied to the far-field boundary, whereas a no-slip condition is used at the solid wall surface.

A moving overlapped Chimera grid system with the near body and the Cartesian off-body grid are employed. Either Cmesh topology grids or O-mesh-based grids are formed, respectively, for the blade and the fuselage. The blade grids extend 1.5 times of a chord length $(c)$ in the normal direction, measured from the blade surface. The cell spacing for the first grid point from the wall boundary used is $1.0 \times 10^{-5} \mathrm{c}$. The off-body grids consist of an inner region that extends $4 c$ upward, $3 c$ below from the blade, and $1.5 c$ away from the blade tip. The far-field boundary is stretched up to $5 R$ (blade radius), centered at the rotor hub. The Cartesian off-body grids have a uniform spacing of $0.1 c$. Figure 2 shows the overall computational grid system used for the HART rotor. The CFD grids result in about 38 million (M) cells in total, $6.4 \mathrm{M}$ for the blade grid, 29.1 M for the off-body grid, and another $2.5 \mathrm{M}$ for the fuselage grid.

3.3. CFD/CSD Coupling Approach. A loose coupling between CAMRAD II and KFLOW codes is adopted for the analysis. The basic idea of the coupling is to benefit the strength of the other code which requires an exchange of information between CSD-computed blade motions and CFD-computed airloads, per revolution base. Figure 3 illustrates a loose coupling strategy. The coupling iteration begins with CSD analysis using a low-fidelity aerodynamic model. The resulting blade motions along with trim control angles are transferred to the CFD code to compute refined aerodynamic forces and moments $(F, M)$. The difference in airloads between the two codes (i.e., delta airloads) is calculated and superposed to the CSD airloads for the updated blade motions and trim controls for the next iteration stage. This process continues until the airloads and trim control angles show a convergence.

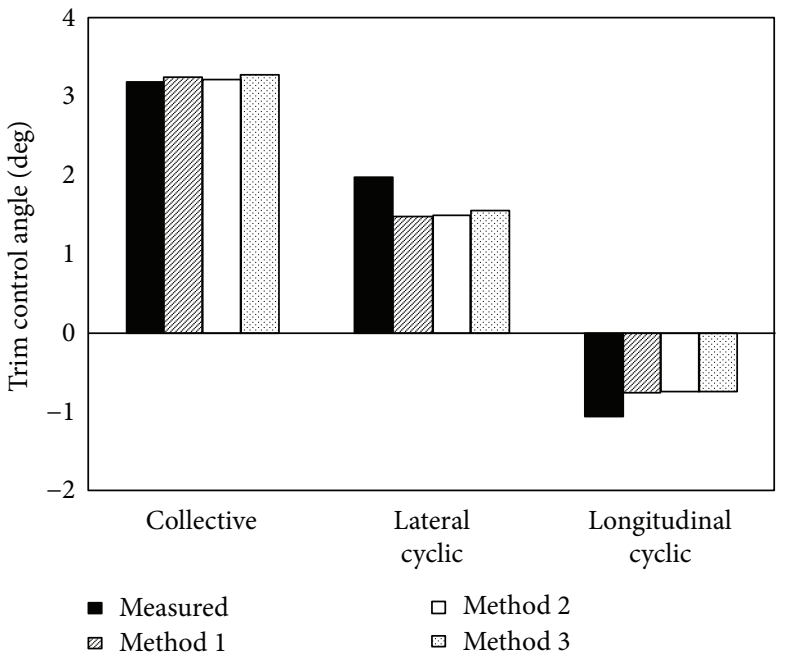

FIgURE 8: Comparison of trim control angles.

The CFD/CSD coupling requires adequate data regression schemes for blade motions and rotor airloads results. The blade motions are interpolated to allow sufficiently smooth curves in both radial and azimuthal directions, as described in van der Wall [19] and Sa et al. [20]. This method is straightforward and based on the best fit of the measured deformations of HART II rotor [12]. In this study, the radial interpolation is represented using polynomials with the seventh order while the time domain is interpolated using a Fourier series up to the eleventh components. The curve-fitted deformation on flap and lag motions is depicted in a $3 \mathrm{D}$ format in Figure 4. The CSD-synthesized data are smooth enough to be taken at any desired stations in a CFD code.

The airloads in spanwise direction are mostly monotonic, and the classical interpolation schemes such as the cubic spline are suitable to fit the data. The timewise airloads signals are arbitrary in general, and no systematic method or standard protocol is set to adopt universally. We employ three different data transfer schemes in association with the time domain airloads: method 1 uses random data selection matched at every azimuth angle of $5 \mathrm{deg}$ for both CFD and CSD analyses; method 2 adopts a low pass filter containing up to $10 / \mathrm{rev}$ (per revolution) for both CFD and CSD data; and method 3 is a mixture of the two methods. The details of the three data synthesis methods for CFD/CSD coupling are summarized in Table 2. Figure 5 illustrates how the data are synthesized for the respective methods considered. As can be seen, the original CFD airloads data represented by the continuous line have an azimuth resolution of $0.2 \mathrm{deg}$ $(\Delta \psi=0.2 \mathrm{deg})$ and appear to contain high-frequency signals (due to BVI events). The dashed line shows a low-passfiltered (up to 10/rev) signal indicating a significant loss of information, particularly for the high-frequency signal. The random data selection made at every $5 \mathrm{deg}$ azimuth angle are represented using hollow diamonds which indicates also some loss of information particularly over highly oscillating data zones. The influence of the candidate data synthesis methods on CFD-/CSD-coupled aeromechanics solutions is examined in the following section. 


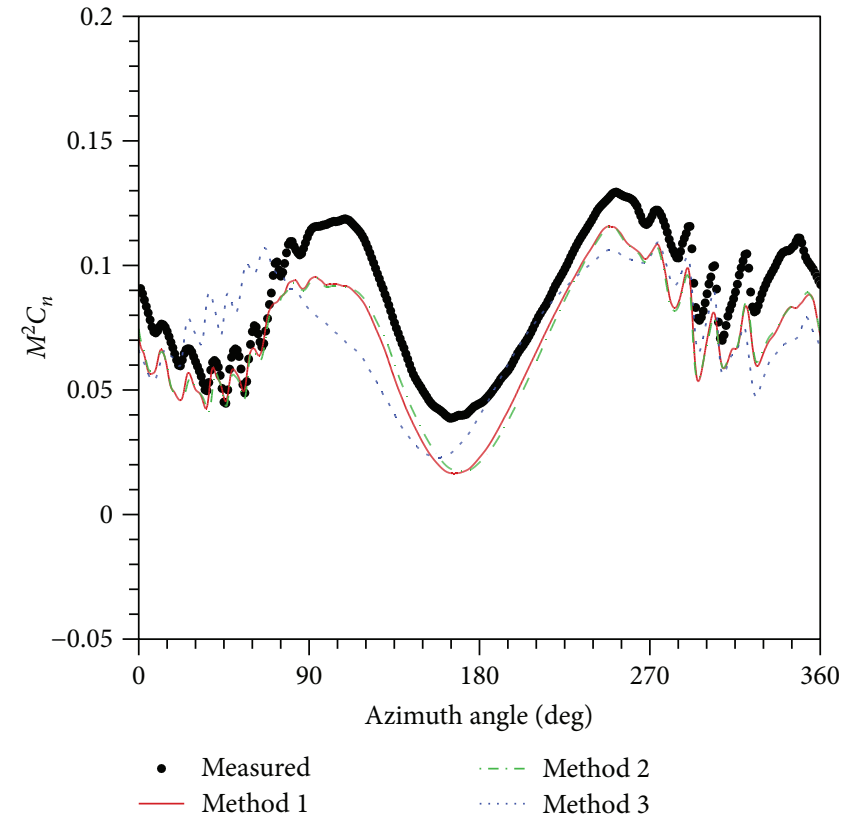

(a) $M^{2} C_{n}$

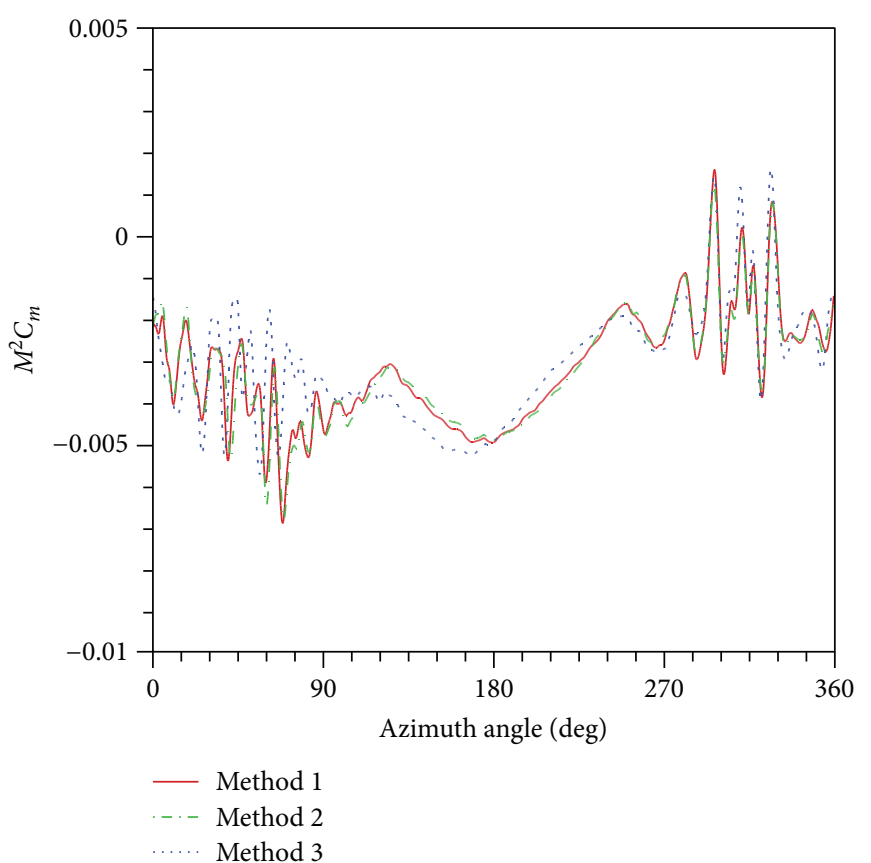

(b) $M^{2} C_{m}$

FIGURE 9: Comparison of section airloads at $87 \%$ radial station.

\section{Results and Discussion}

The three data transfer schemes are investigated first to evaluate the validity and overall performance of the techniques with respect to the coupling. To this end, the measured data of HART rotor are used as the referendum to evaluate the accuracy of the coupled predictions according to the data transfer methods. Next, the present CFD-/CSD-coupled results using the desired transfer method are compared with the measured data and also with CSD-alone predictions. Only the baseline (BL; Dpt. 140) condition of HART rotor is considered. It is noted that a pitch-bearing stiffness of $1706 \mathrm{~N}-\mathrm{m} / \mathrm{rad}$ is used to match the first torsion frequency and to represent the control system characteristic of HART rotor [21].

\subsection{Parametric Investigation of Data Transfer Methods.} Following the procedures given in Figure 3, the CFD-/CSDcoupled trim iterations are marched using the methods proposed in this study. The trim is handled by CSD code to match the trim targets specified as $3100 \mathrm{~N}, 11.2 \mathrm{~N}-\mathrm{m}$, and $-20 \mathrm{~N}-\mathrm{m}$, respectively, for thrust, roll, and pitching moment. Figures 6 and 7 show the convergence behavior of CFD airloads $M^{2} C_{n}$ (Mach number-scaled section normal forces) and the corresponding delta airloads with coupling iterations, respectively, for the three data transfer methods. It is clearly indicated that all methods lead to a convergence after 6 to 7 coupling iterations are stepped. The obvious differences according to the data transfer methods are represented in delta airloads results (Figure 7). In methods 1 and 3, some of the BVI peaks are preserved and passed over to the next coupling iterations while no such peaks are inherited to the next cycle in method 2 because of the low-pass-filtering operation performed at each step. It should be remarked that, in method 3, the BVI oscillatory peaks in the first and fourth quadrants of the rotor are gaining strengths to become very strong spikes as the coupling iterations are stepped further, in comparison with the other methods. Figure 8 shows the predicted control trim angles according to the three methods, as compared with the measured data. All methods indicate good correlation for the collective pitch settings with a significant underprediction in the cyclic pitch angles by up to $28.5 \%$. However, there appear no remarkable deviations on predicted trim control angles between the data transfer methods.

Figure 9 shows the comparison of converged CFD airloads results on section normal forces and pitching moments, respectively, at $87 \%$ radial station of the rotor. Since no measured pitching moments are available for HART rotor, only the predicted results are compared with each other. As can be seen in both plots, methods 1 and 2 indicate nearly identical results leading to good agreements with the measured data. The peak-to-peak intensity of the airloads signal along with the down-up pattern near the front edge of the rotor disk is captured correctly. In addition, both BVI events apparent in the first and fourth quadrants of the rotor disk are predicted nicely in terms of magnitudes and phases of the signals. However, method 3 indicates significant deviations for section normal forces and section pitching moments over most of the azimuthal domain, as compared with the other methods, resulting in a considerable offset with the measured data. For the section normal forces, the predicted mean in the first quadrant increases significantly and the down-up pulse leads the measured data by about $15 \mathrm{deg}$. The BVI peaks in the pitching moment signal increase somewhat also. The higher BVI peak magnitudes of method 3 are 


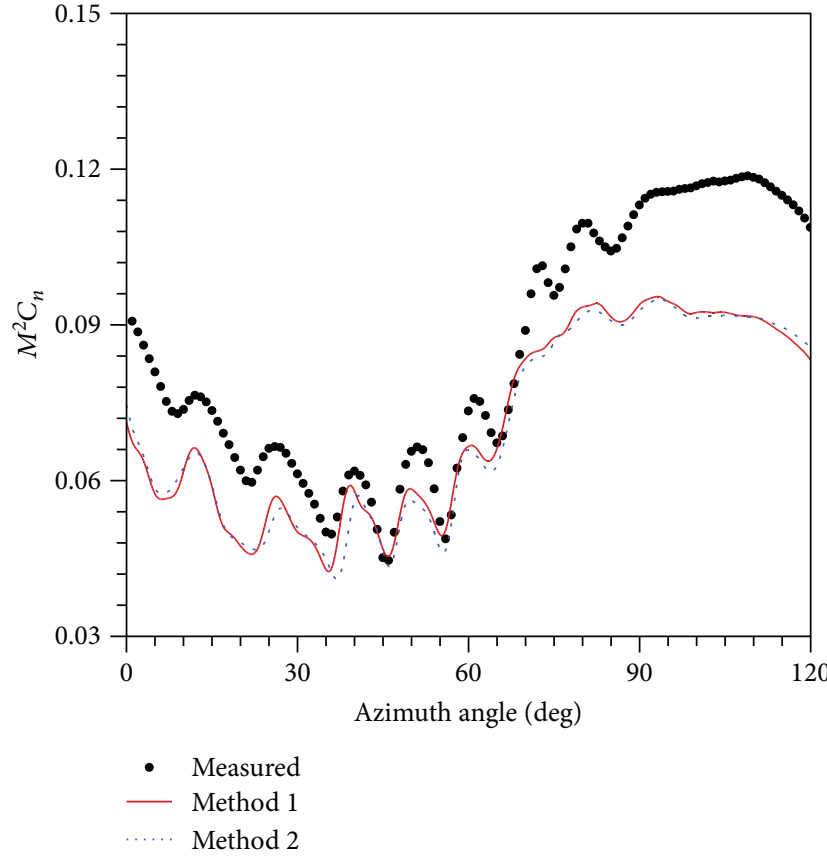

(a) Advancing side

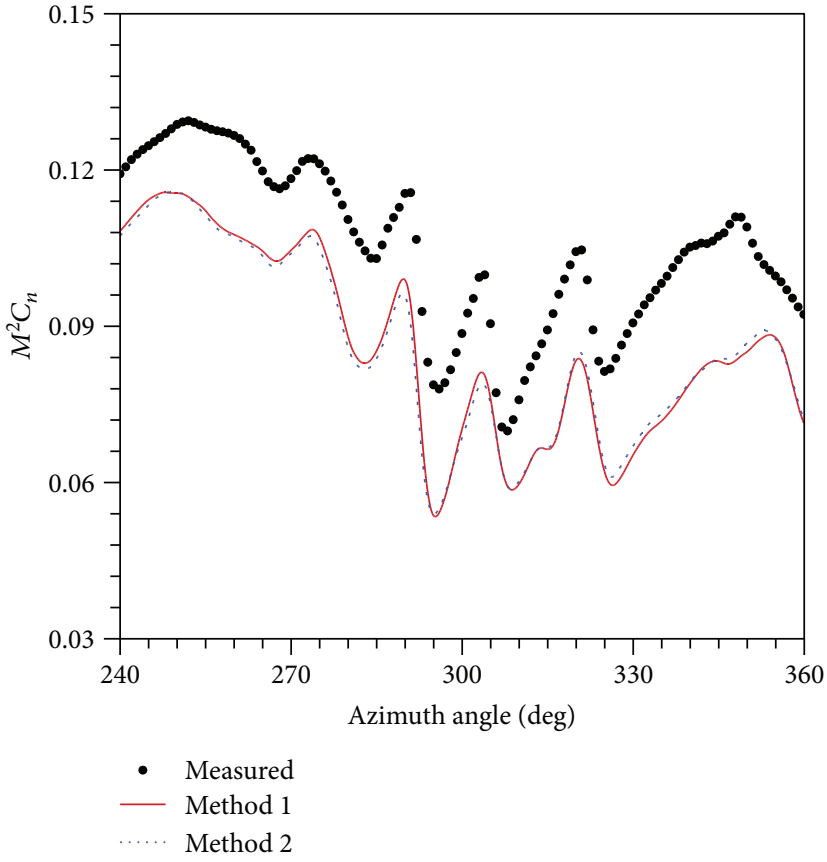

(b) Retreating side

Figure 10: Comparison of enlarged view of section airloads $M^{2} C_{n}$ at $87 \%$ radial station.

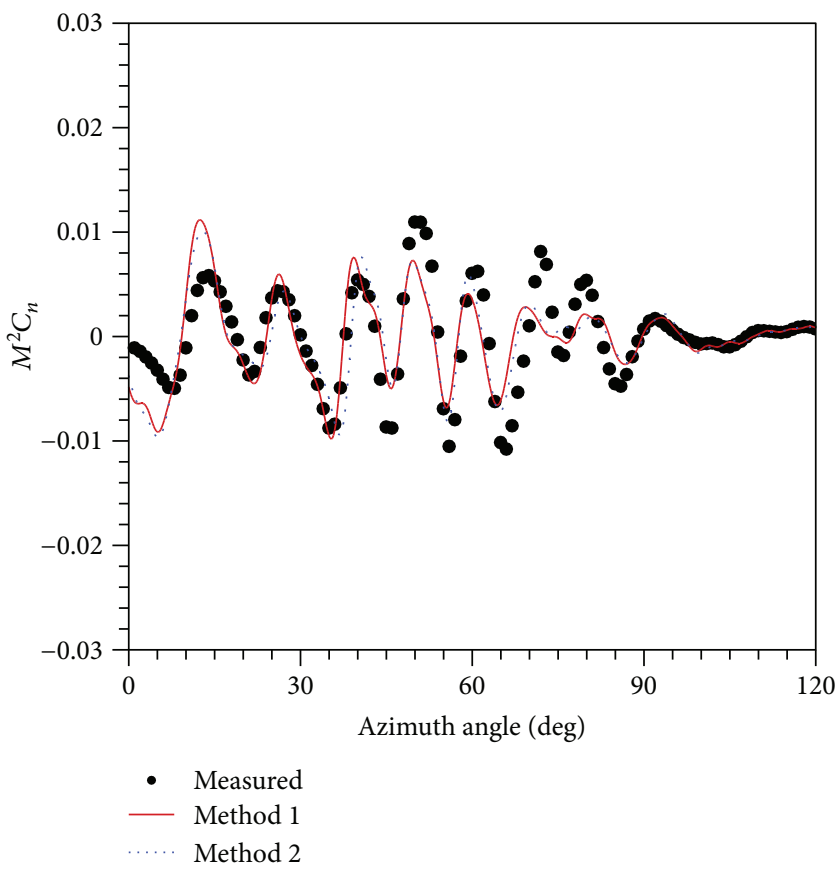

(a) Advancing side

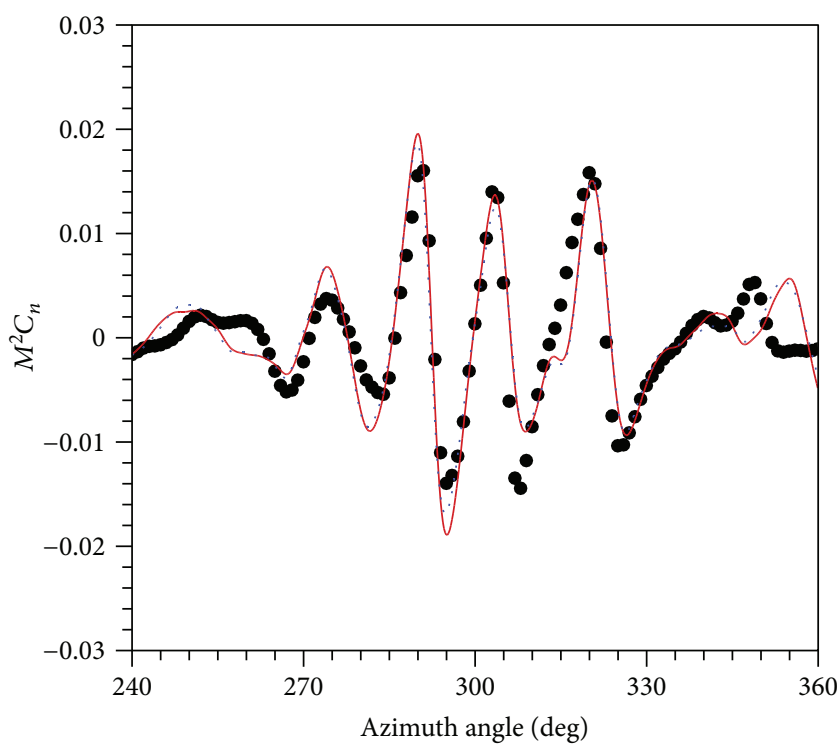

- Measured

- Method 1 Method 2

(b) Retreating side

FIGURE 11: Comparison of higher harmonic contents (11/rev and higher) of section airloads $M^{2} C_{n}$ at $87 \%$ radial station.

due to an increase in peaks accumulated during the coupling iterations before the convergence is reached, as observed in Figure 7. This can also be explained considering the way the coupling computations are managed. It is recalled that method 2 is a mixture method since CFD airloads are interpolated in the same way adopted in method 1 (i.e., random selection at every azimuth angle of $5 \mathrm{deg}$ ), and CSD airloads are low-pass filtered as method 2. A natural consequence of the mixed method is that the differences between CFD and CSD airloads (i.e., delta airloads) may be gaining in strengths, particularly for the case when highly oscillatory signals (larger than 10/rev) are present. More specifically, some of the spikes and kinks existed in the original signal can be survived after the random selection (method 1) 


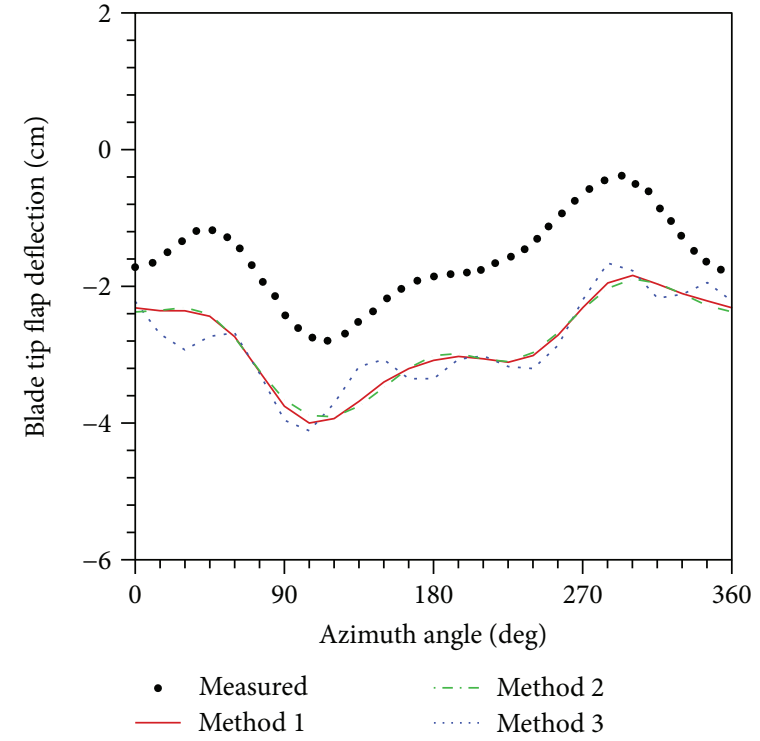

(a) Flap motion

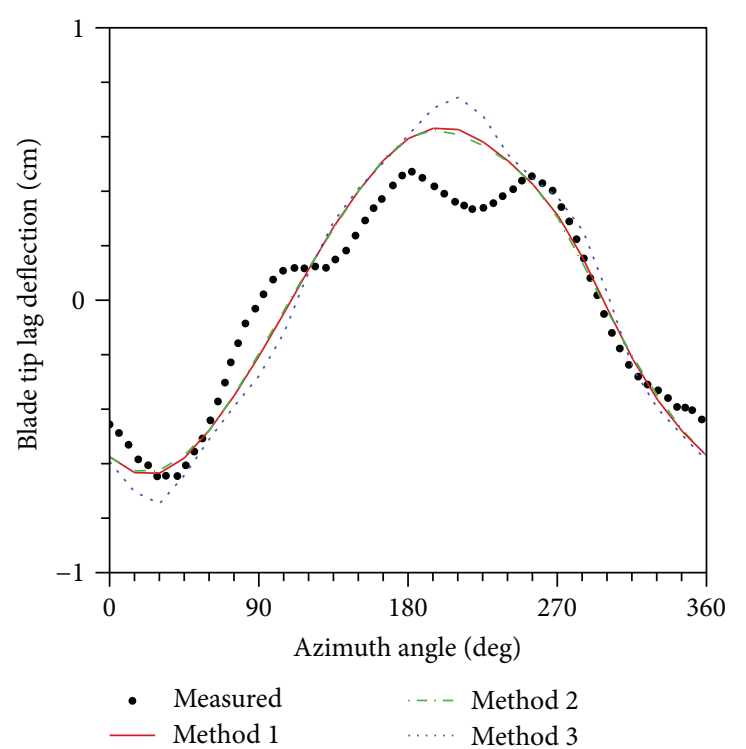

(b) Lag motion (mean removed)

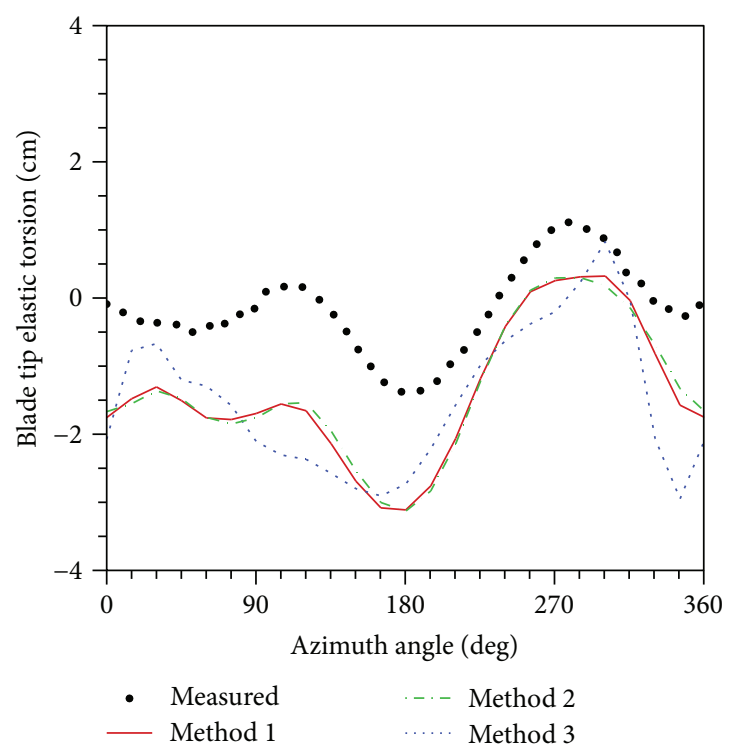

(c) Torsion motion

FIgURE 12: Comparison of blade tip elastic deflections.

combined with the smoothening process (method 2), and these could be tossed to the consecutive coupling cycles for more intensified oscillations (which is nonphysical), as is observed in Figure 7(b).

Besides the mixture case, method 1 or method 2 indicates reasonable correlation with the measured data. For clarity, enlarged views on the advancing and retreating sides of the section normal forces predicted at $87 \%$ rotor radial station are compared against the measured data in Figure 10. Most of the BVI events are seen to be predicted correctly by method 1 or method 2 , in terms of the number of peak oscillation counts, peak-to-peak magnitudes, and phases of the measured signals. It should be mentioned that both methods indicate no significant deviations on the airloads predictions, despite the fundamental differences in interpolating the data.
Figure 11 presents the comparison of higher harmonic components of the section airloads $M^{2} C_{n}$ after removing up to the lowest $10 /$ rev harmonic contents. Once again, both results by methods 1 and 2 show no noticeable deviations with each other. The predicted phase responses show excellent correlation against the measured data with underpredictions in magnitudes around the azimuth angle of $60 \mathrm{deg}$. The elastic deformations for flap, lag, and torsion predicted at the blade tip by the three data transfer methods are compared with the measured data in Figure 12. Similar to the results found on airloads, both methods 1 and 2 show nearly identical blade motion results, whereas more oscillatory response with higher harmonics is seen in method 3 which should be caused by the stronger peaks combined with the varied mean of the airloads predictions. Based on the observations, 


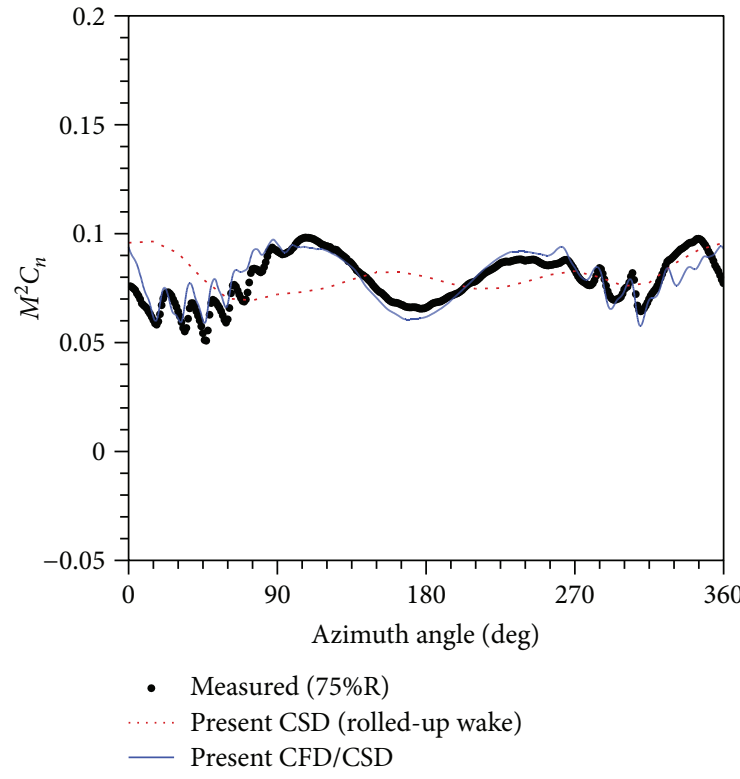

(a) $r / R=0.75$

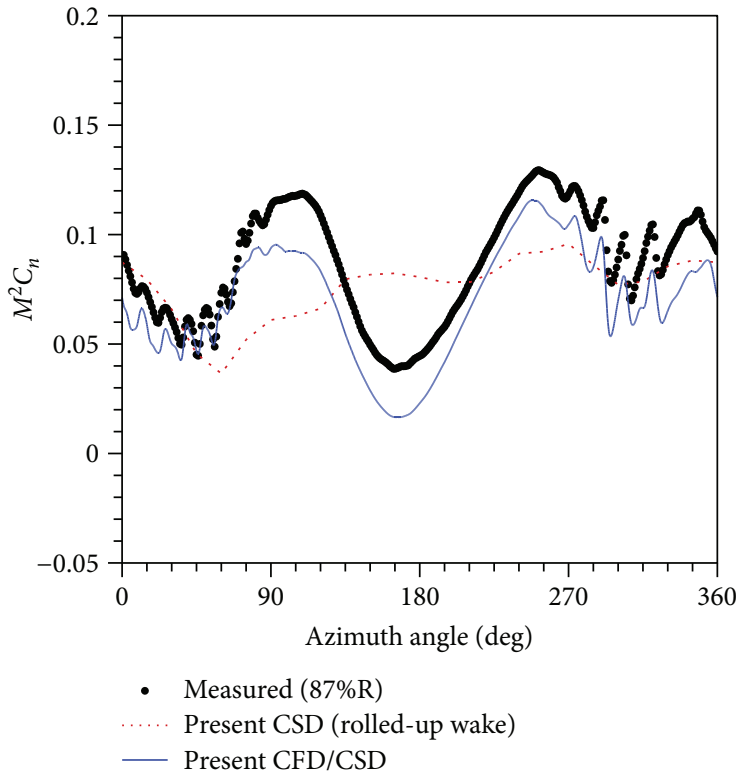

(b) $r / R=0.87$

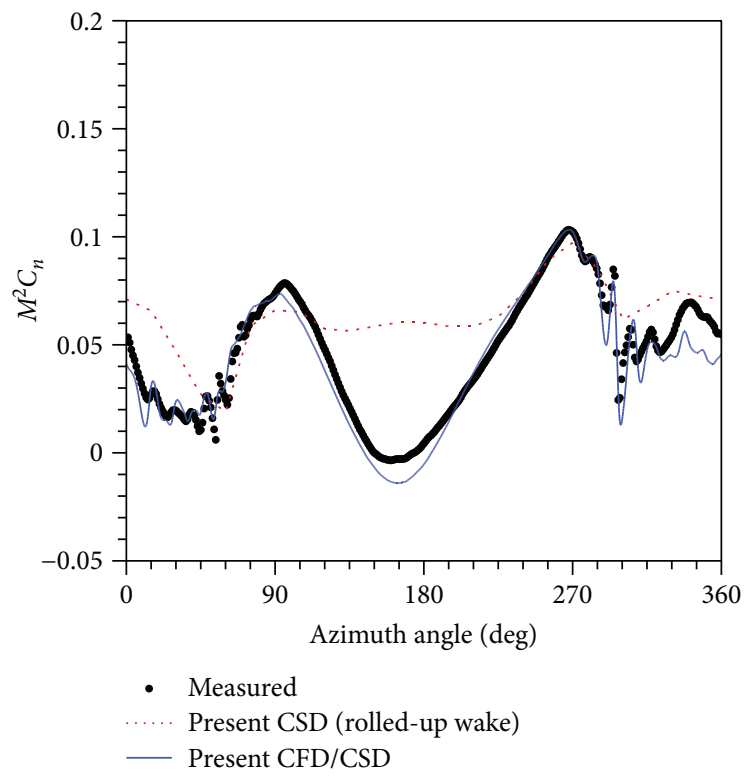

(c) $r / R=0.97$

FIGURE 13: Correlation of section normal forces $M^{2} C_{n}$ with radial stations.

method 1 is adopted in synthesizing the data for CFD/CSD coupling for the validation of the measured HART data, which will be presented in the next section.

4.2. Validation of HART Rotor. The measured airloads data of HART rotor in descent at different radial locations $(r / R=0.75,0.87$, and 0.97$)$ are compared in Figure 13, against the predicted results by CFD/CSD coupling and CSD approach with a rolled-up free-wake model. The free-wake representation is modeled using an initial core size of $0.5 c$ with a square root growth over four rotor revolutions. The timewise resolution has $15 \mathrm{deg}$ in the CSD analysis. The CSD predictions on section airloads show only fair correlations with the measured data. Specifically, the typical down- up pattern seen near the front edge of the rotor disk in the measured signal is missed wholly. The CFD/CSD coupling improves the correlation significantly to match the measured data in terms of both waveforms and amplitudes. The means are predicted accurately by CFD/CSD coupling particularly at $r / R=0.75$ and 0.97 with a slight underestimation at $r / R=0.87$ as compared to the measured data. It should be mentioned that the correct predictions of phase response as well as the improved BVI signals in the advancing side are due to the incorporation of the fuselage model in the CFD/CSD approach, as observed previously by Jung et al. [14]. The vibratory airloads components keeping 3/rev and higher are compared next in Figure 14 according to the three radial stations. The vibratory components are useful 


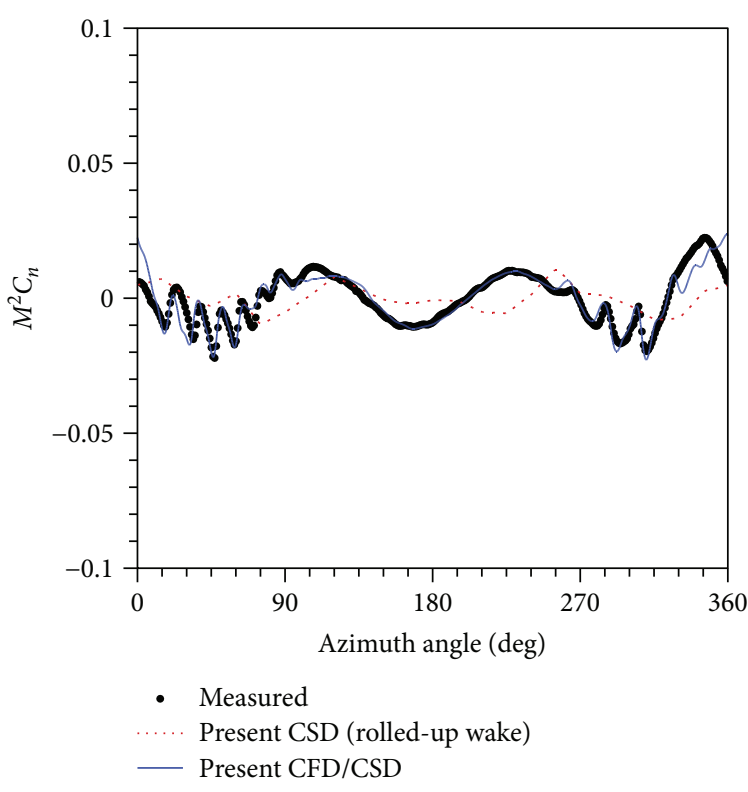

(a) $r / R=0.75$

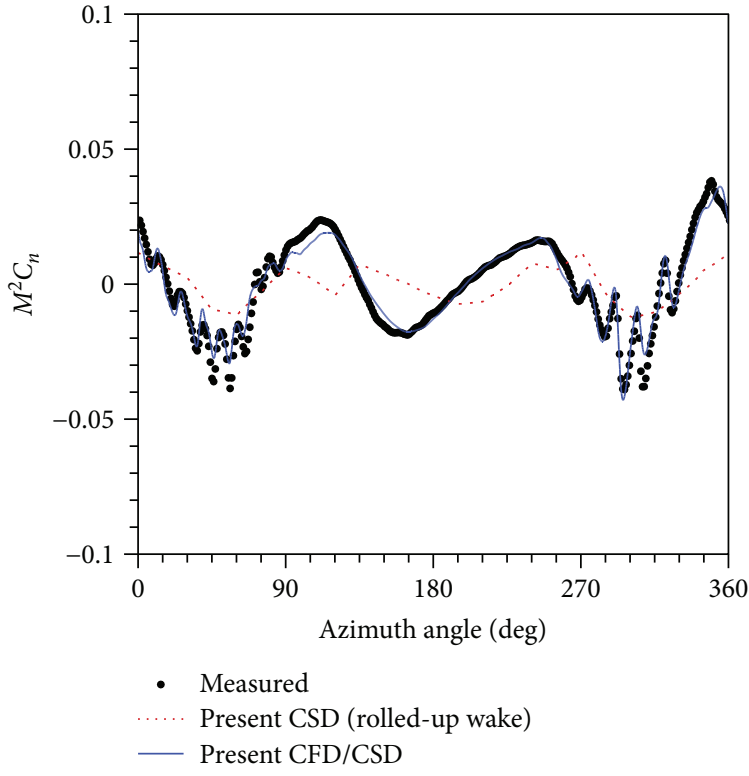

(b) $r / R=0.87$

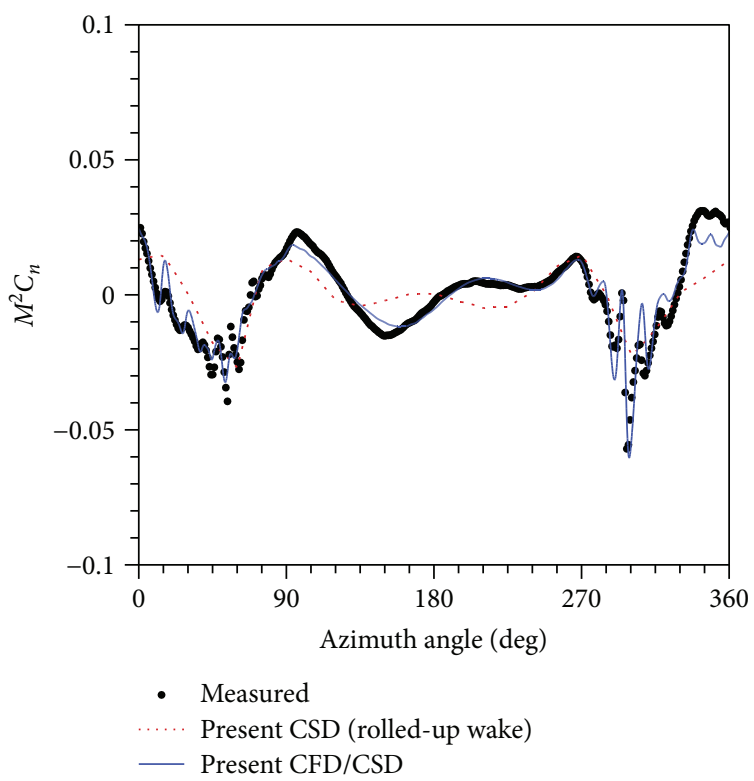

(c) $r / R=0.97$

Figure 14: Correlation of vibratory components (3/rev and higher) of $M^{2} C_{n}$ with radial stations.

to determine the hub vibration characteristic of a rotor with finite number of blades. As can be seen, the predicted airloads exhibit excellent correlations with the measured data particularly on BVI oscillatory peaks in advancing and retreating sides, down-up pattern near the front edge, and peak-to-peak magnitudes and phases of the timewise airloads signal. It is noted again that the CSD results show limited prediction capability in the computation of vibratory airloads components.

To gain more insights on BVI airloads, the gradient of the section normal forces with respect to the time, $d\left(M^{2} C_{n}\right) / d \psi$, is investigated in Figure 15. The timewise gradient signals are closely related with the acoustic noise emission since the sound radiation involves the partial derivative of air loading (acoustic pressure) with time. The predicted CSD results miss all BVI events in rotor advancing and retreating sides due to the limited modeling capability of its own. The CFD-/CSD-coupled predictions indicate good agreements on the number of BVI counts and the phases while some of peak amplitudes are underestimated compared to the measured data. In predicting BVI peak strengths, the correlation is generally excellent in the retreating side and less satisfactory in the advancing side. The neglect of hub model as well as the limitation of RANS model in approximating the flow equations for such a highly fluctuating BVI event is the possible source of the discrepancy. Despite the limits, however, the computed CFD/CSD results demonstrate the current level of prediction for HART rotor. 


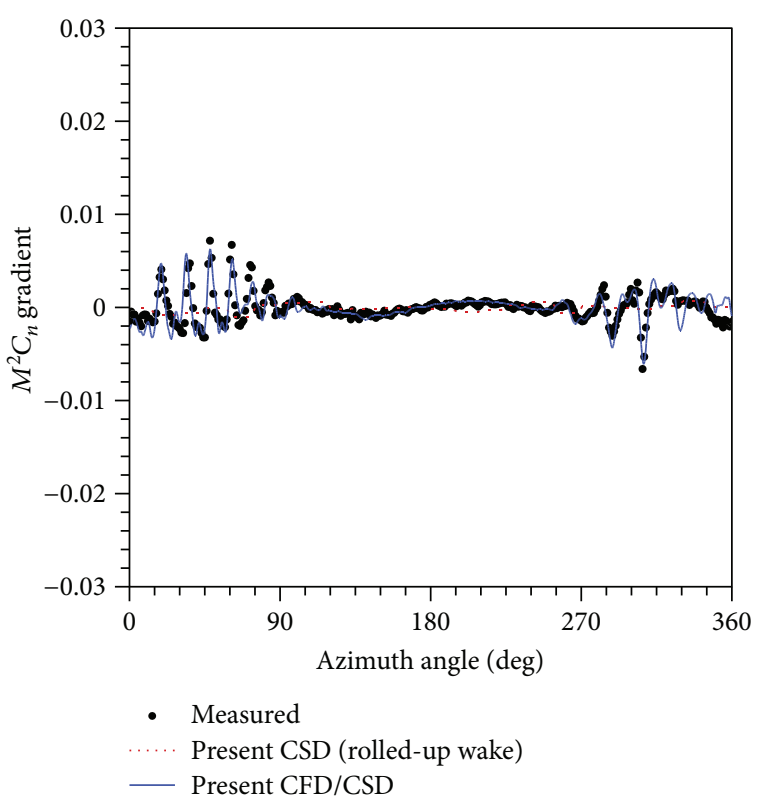

(a) $r / R=0.75$

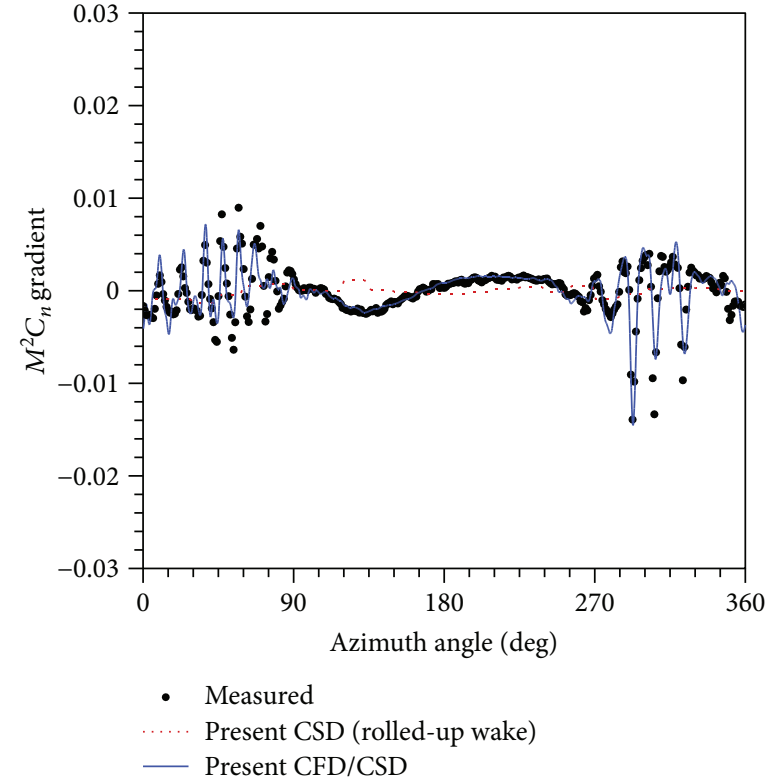

(b) $r / R=0.87$

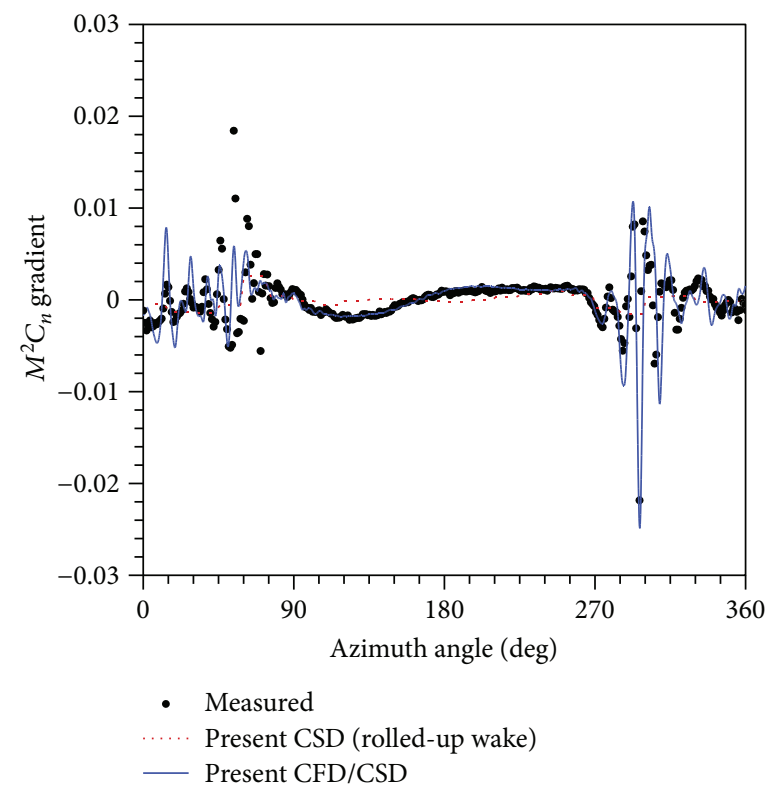

(c) $r / R=0.97$

Figure 15: Correlation of the gradient of section normal forces $d\left(\mathrm{M}^{2} C_{n}\right) / d \psi$ with radial stations.

The predicted blade tip elastic deformations on flap, lag, and torsion motions are compared with the measured data in Figure 16. Other than the previous airloads comparison, less satisfactory correlation is obtained by CFD/CSD predictions while comparable results are obtained by CSD approach at least in terms of the mean response. However, both the waveform and the phase response are better predicted by CFD/CSD coupling. Specially, the convex pattern around the azimuth angles of $180 \mathrm{deg}$ in the measured flap motion as well as an up-down pattern seen in the second quadrant of the measured torsion is captured nicely with the CFD/CSD coupling. Despite the improved predictions with the CFD/CSD coupling, there remains an apparent offset on flap and twist deformations between CFD/CSD predictions and the measured data. One possible reason of the discrepancy is the blade-to-blade dissimilarity of HART rotor. It should be mentioned that the instrumented blade (number 1 blade) is reported to be heavier by about $6 \%$ than the rest of the blades [16]. In addition, the blade property measurement has not been made with the instrumented blade (H1Y) but using number 3 blade (H1B) and a spare HART blade (H1S) [15]. In addition, sophisticated RTB (rotor track and balance) process is skipped during the HART measurement campaign. Even though no measurement record is found for displacements of the individual HART blades, one can estimate the order of magnitudes 


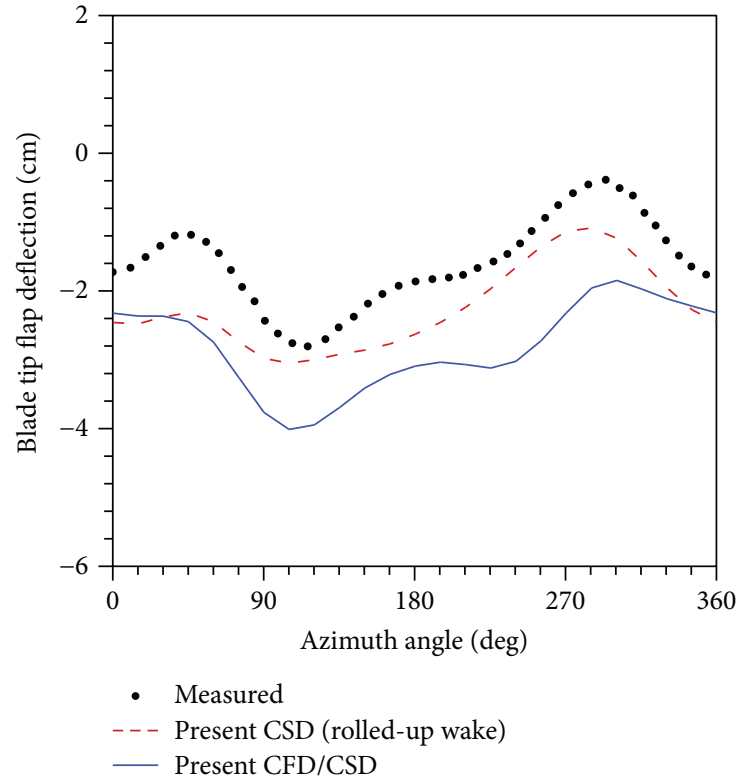

(a) Flap motion

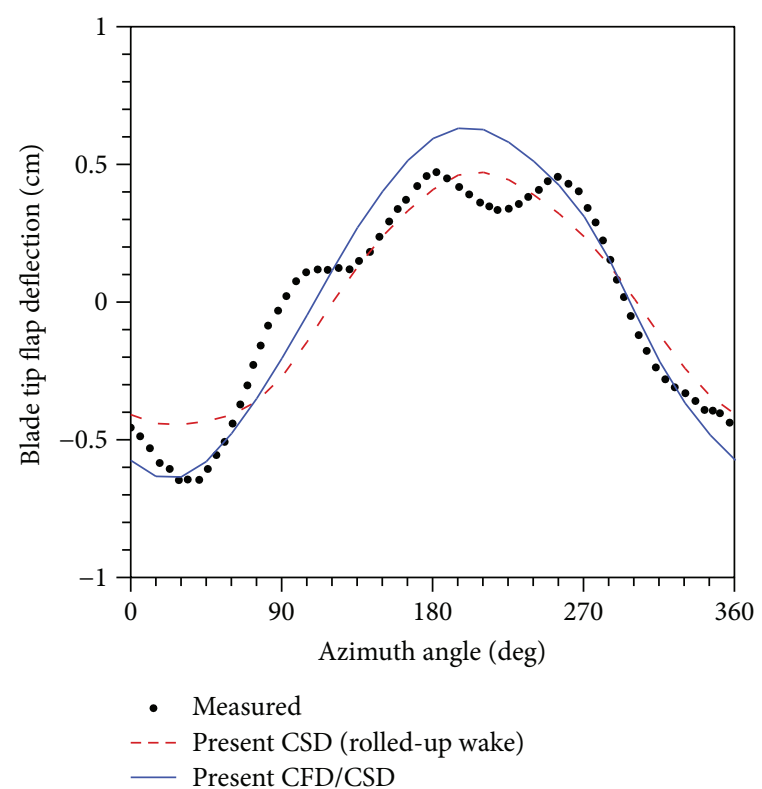

(b) Lag motion (mean removed)

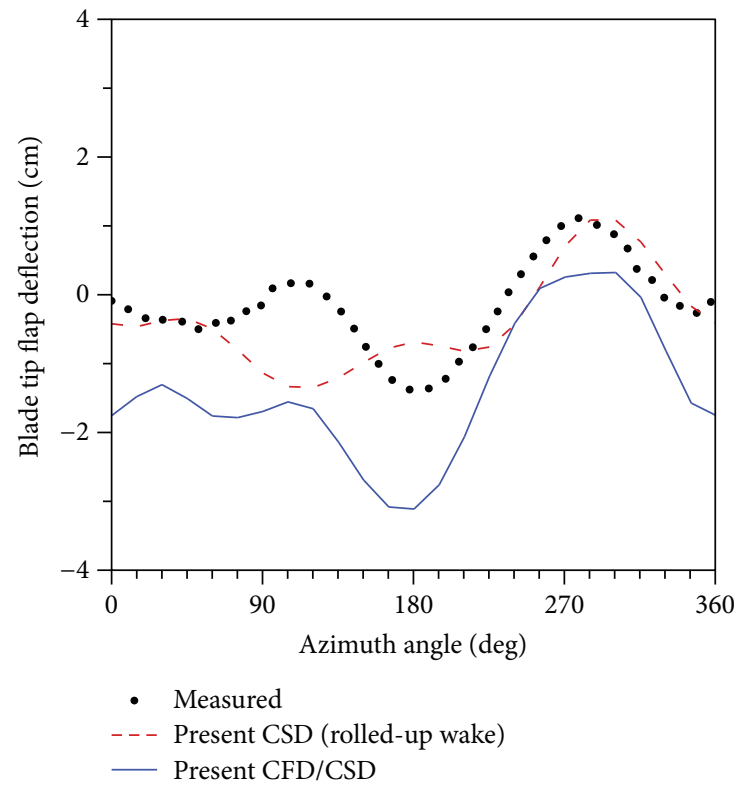

(c) Torsion motion

FIgURE 16: Correlation of blade tip elastic deflections.

considering HART II data. It is found that the maximum deviation between the blades of HART II rotor is about $2 \%$ for the tip flap deflections [12]. Taking these facts into account, reasonable agreements appear to be reached using the present CFD/CSD predictions. Finally, in Figure 17, the structural moments at a blade inboard station $r / R=0.144$ are compared between the present CFD/CSD results, CSD predictions, and the measured data. The mean is removed for comparison. Fair-to-good correlation is observed with the present predictions. The CFD/CSD coupling improves the correlation significantly in terms of the peak-to-peak amplitudes and the waveforms of the measured flap bending and torsion moment signals. The phase response in the retreating side of the flap bending moment as well as the torsion moment in the second quadrant is better predicted using the CFD/CSD coupling analysis than the CSD approach, even though a phase lead is observed in the predicted flap bending moments as compared with the measured data. Use of the blade structural properties other than the instrumented blade may cause the error in the predicted results.

\section{Conclusions}

In this work, a numerical study is performed for the validation of measured HART data as well as the validity of data 


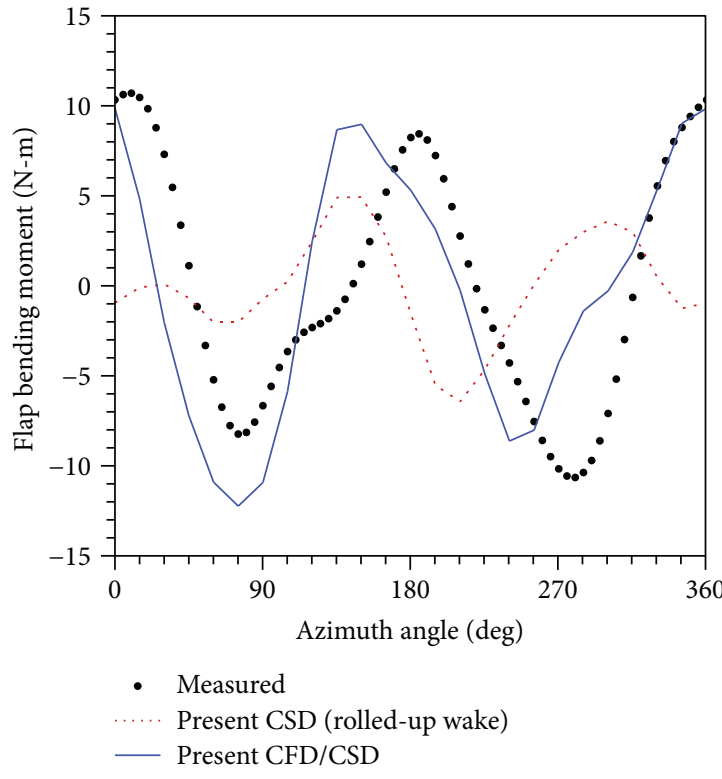

(a) Flap bending

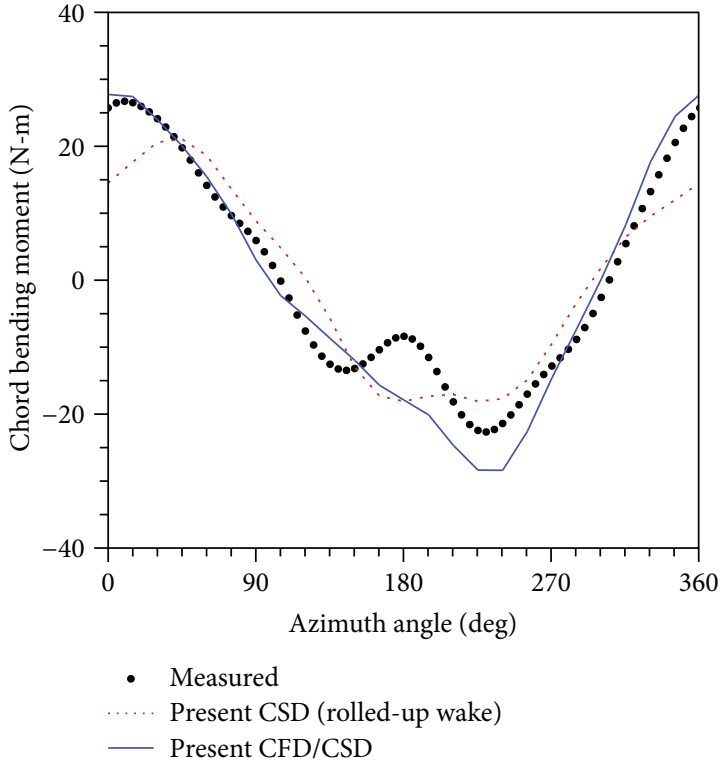

(b) Chord bending

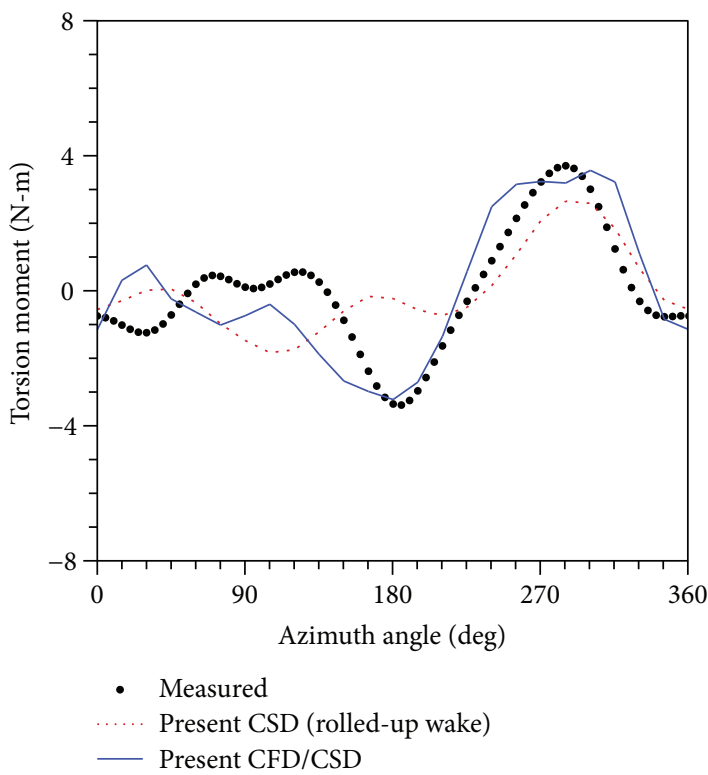

(c) Torsion moment

Figure 17: Correlation of structural moments at a blade inboard station $r / R=0.144$ (mean removed).

transfer methods using CFD-/CSD-coupled approach. The data transfer methods include a random data selection matched with the timewise resolution of CSD analysis and/ or a low-pass harmonic filter, leading to three different methods (methods 1 to 3). A 3D compressible RANS flow solver KFOW is coupled with a CSD analysis code CAMRAD II for this purpose. Based on the observations, the following conclusions are drawn:

(1) Good convergence characteristic is reached on airloads and delta airloads for all the data transfer methods after six to seven coupling iteration cycles are marched. This verifies the robustness of the delta airloads technique in CFD-/CSD-coupled approach.
(2) In both CFD and CSD analyses, any consistent use of data synthesis techniques (e.g., method 1 or 2 ) is desired to benefit the coupling approach. A mixture rule (method 3 ) should be prohibited especially when high-frequency signals such as BVI oscillations are present in the airloads data. It is found that the delta airloads become more oscillatory as some of the kinks and spikes existed in airloads data get survived and intensified in strengths with the coupling iterations, leading to a poor correlation for airloads and blade elastic motions as compared with the measured data.

(3) The data transfer scheme either method 1 or method 2 demonstrates nearly identical results on airloads 
and elastic blade motions despite the differences in processing the airloads data. Either method leads to good agreements with the measured HART data. The incorporation of the fuselage model in the CFD analysis contributes to the close correlation, particularly on the phase response around the front edge of the rotor and improved BVI predictions in the advancing side of the rotor disk.

(4) The present CFD/CSD coupling shows good-toexcellent agreements on airloads as well as the timewise gradient signals $d\left(M^{2} C_{n}\right) / d \psi$ of HART rotor obtained at the three measurement stations distributed along the blade. The number of BVI oscillatory events and the phase response of the airloads signals are accurately captured with the CFD-/CSD-coupling approach while underestimating some of the gradient signals of BVI peaks in the advancing side of the rotor. The neglect of hub model as well as the use of RANS model in approximating the flow equations around the rotor is the possible source of the discrepancy for such a highly fluctuating BVI event.

\section{Conflicts of Interest}

The authors declare that there are no conflicts of interest regarding the publication of this paper.

\section{Acknowledgments}

This work was supported by the KEIT Research grant of 2016 (no. 10053155). This paper resulted from the Konkuk University research support program. The authors thank HART team for the test data.

\section{References}

[1] C. Tung, F. X. Caradonna, and W. Johnson, "The prediction of transonic flows on an advancing rotor," Journal of the American Helicopter Society, vol. 31, no. 4, pp. 4-11, 1986.

[2] P. Beaumier, "A coupling procedure between a rotor dynamics code and a 3D unsteady full potential code," in American Helicopter Society Aeromechanics Specialists' Conference, pp. 19-21, San Francisco, CA, USA, 1994.

[3] M. Potsdam, H. Yeo, and W. Johnson, "Rotor airloads prediction using loose aerodynamic/structural coupling," Journal of Aircraft, vol. 43, no. 3, pp. 732-742, 2006.

[4] Y. H. Yu, B. Gmelin, H. Heller, J. J. Philippe, E. Mercker, and J. S. Preisser, "HHC aeroacoustics rotor test at the DNW the joint German/French/US HART project," in Proceedings of the 20th European Rotorcraft Forum, pp. 4-7, Amsterdam, Netherlands, 1994.

[5] W. Johnson, "Milestones in rotorcraft aeromechanics Alexander A. Nikolsky honorary lecture," Journal of the American Helicopter Society, vol. 56, no. 3, pp. 1-24, 2011.

[6] Y. H. Yu, C. Tung, B. G. van der Wall et al., "The HART-II test: rotor wakes and aeroacoustics with higher-harmonic pitch control (HHC) inputs - the joint German/French/Dutch/US project," in American Helicopter Society 58th Annual Forum Proceedings, pp. 11-13, Montreal, Canada, 2002.
[7] C. Tung, J. M. Gallman, R. Kube et al., "Prediction and measurement of blade - vortex interaction loading," in 1st CEAS/AIAA Aeroacoustics Conference Proceedings, pp. 12 15, Munich, Germany, 1995.

[8] J. W. Lim, Y. H. Yu, and W. Johnson, "Calculation of rotor blade-vortex interaction airloads using a multiple-trailer free-wake model," Journal of Aircraft, vol. 40, no. 6, pp. 1123-1130, 2003.

[9] J. W. Lim, T. A. Nygaard, R. Strawn, and M. Potsdam, "Bladevortex interaction airloads prediction using coupled computational fluid and structural dynamics," Journal of the American Helicopter Society, vol. 52, no. 4, pp. 318-328, 2007.

[10] H. Yeo and W. Johnson, "Assessment of comprehensive analysis calculation of airloads on helicopter rotors," Journal of Aircraft, vol. 42, no. 5, pp. 1218-1228, 2005.

[11] H. Yeo and W. Johnson, "Prediction of rotor structural loads with comprehensive analysis," Journal of the American Helicopter Society, vol. 53, no. 2, pp. 193-209, 2008.

[12] B. G. van der Wall, J. W. Lim, M. J. Smith et al., “The HART II international workshop: an assessment of the state-of-the-art in comprehensive code prediction," CEAS Aeronautical Journal, vol. 4, no. 3, pp. 223-252, 2013.

[13] M. J. Smith, J. W. Lim, B. G. van der Wall et al., "The HART II international workshop: an assessment of the state of the art in CFD/CSD prediction," CEAS Aeronautical Journal, vol. 4, no. 4, pp. 345-372, 2013.

[14] S. N. Jung, J. H. Sa, Y. H. You, J. S. Park, and S. H. Park, "Loose fluid-structure coupled approach for a rotor in descent incorporating fuselage effects," Journal of Aircraft, vol. 50, no. 4, pp. 1016-1026, 2013.

[15] S. N. Jung and B. H. Lau, Determination of HART I Blade Structural Properties by Laboratory Testing, 2012, NASA CR 2012-216039.

[16] S. N. Jung, Y. H. You, B. H. Lau, W. Johnson, and J. Lim, "Evaluation of rotor structural and aerodynamic loads using measured blade properties," Journal of the American Helicopter Society, vol. 58, no. 4, 2013.

[17] W. Johnson, CAMRAD II: Comprehensive Analytical Model of Rotorcraft Aerodynamics and Dynamics, Johnson Aeronautics, Palo Alto, CA, USA, 1992.

[18] J. W. Kim, S. H. Park, and Y. H. Yu, "Euler and Navier-Stokes simulations of helicopter rotor blade in forward flight using an overlapped grid solver," in 19th AIAA CFD Conference, San Antonio, TX, 2009.

[19] B. G. van der Wall, Mode Identification and Data Synthesis of HART II Blade Deflection Data, Institute Report, IB-1112007/28, Braunschweig, 2007.

[20] J. H. Sa, J. W. Kim, S. H. Park, J. S. Park, S. N. Jung, and Y. H. $\mathrm{Yu}$, "KFLOW results of airloads on HART-II rotor blades with prescribed blade deformation," International Journal of Aeronautical and Space Sciences, vol. 10, no. 2, pp. 52-62, 2009.

[21] D. H. Na, Y. H. You, and S. N. Jung, "Comprehensive aeromechanics predictions on air and structural loads of HART I rotor," International Journal of Aeronautical \& Space Sciences, vol. 18, no. 1, pp. 165-173, 2017. 


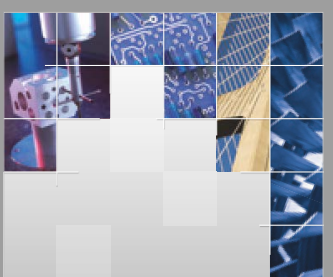

\section{Enfincering}
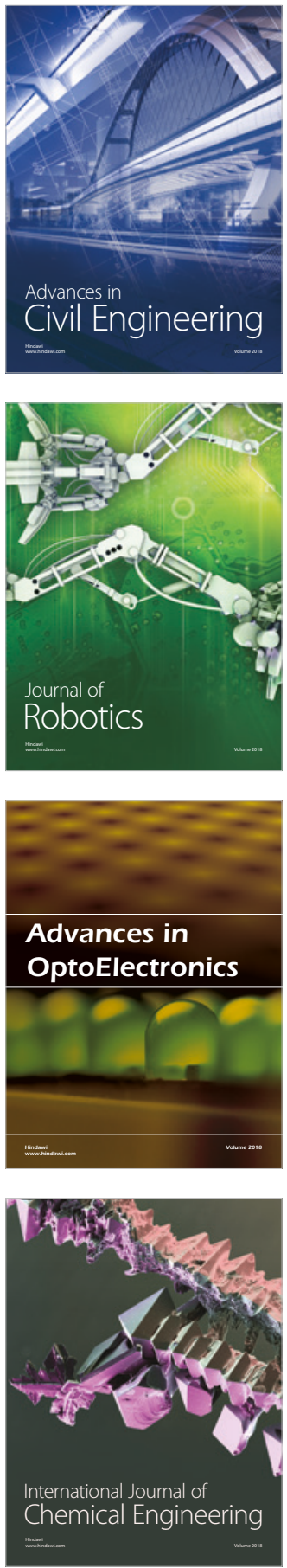

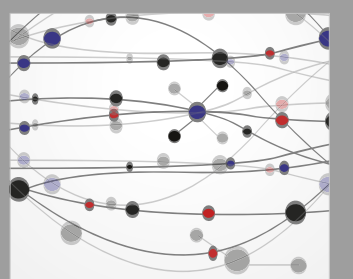

\section{Rotating \\ Machinery}

The Scientific World Journal

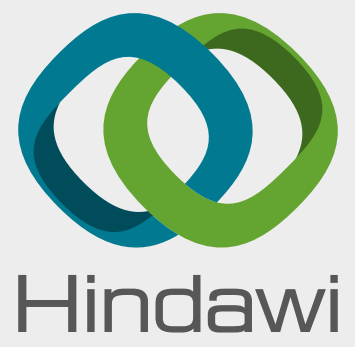

Submit your manuscripts at

www.hindawi.com
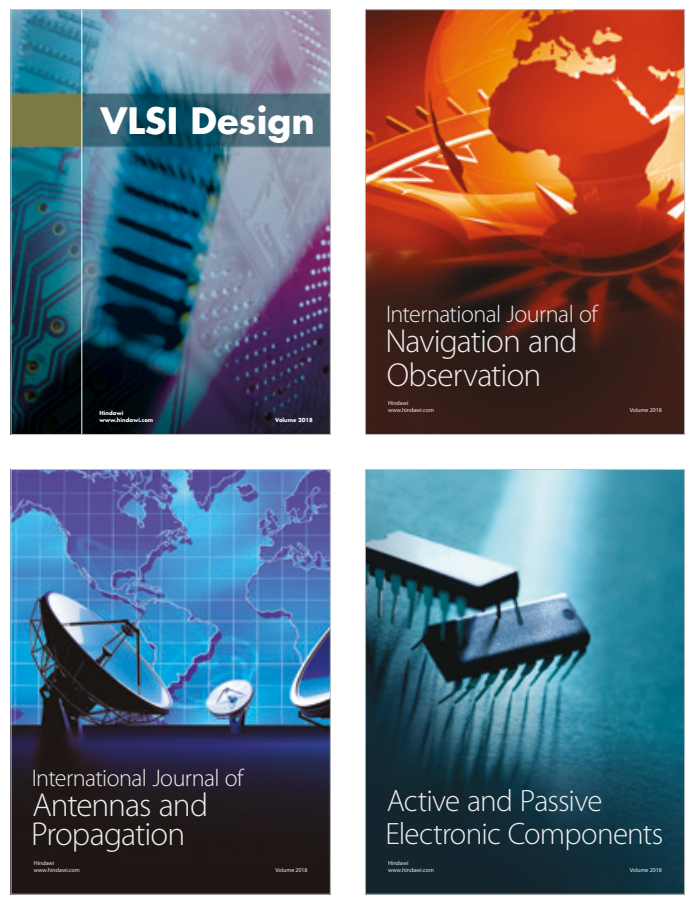
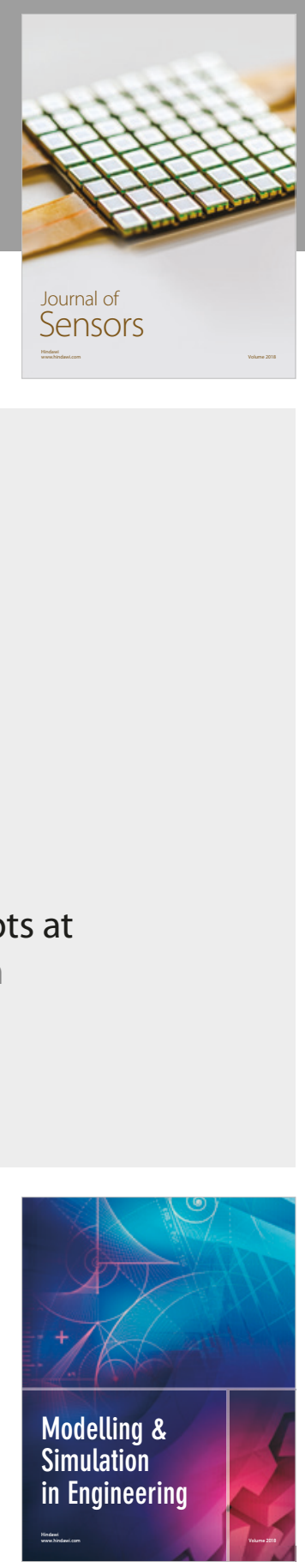

\section{Advances \\ Multimedia}
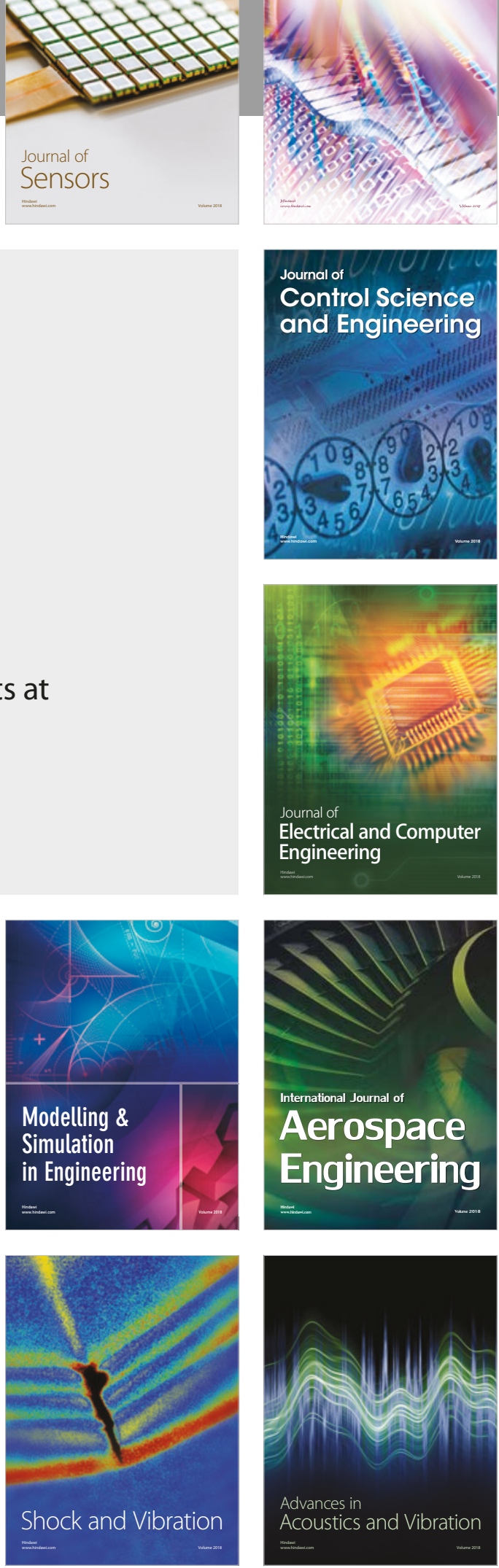